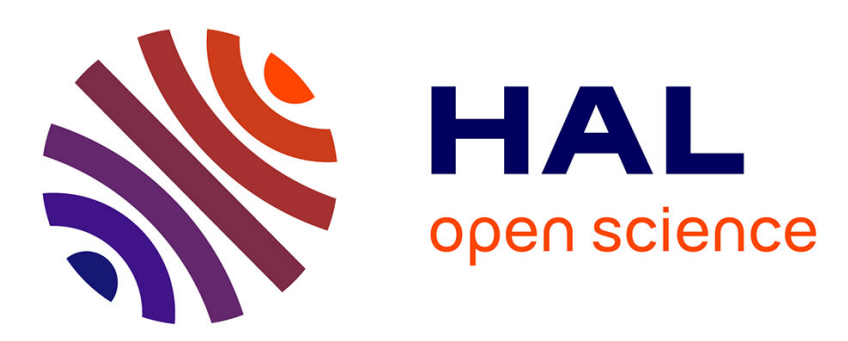

\title{
Direct numerical simulation of the impact of a droplet onto a hot surface above the Leidenfrost temperature
}

Lucia Rueda Villegas, Sébastien Tanguy, Guillaume Castanet, Ophélie Caballina, Fabrice Lemoine

\section{- To cite this version:}

Lucia Rueda Villegas, Sébastien Tanguy, Guillaume Castanet, Ophélie Caballina, Fabrice Lemoine. Direct numerical simulation of the impact of a droplet onto a hot surface above the Leidenfrost temperature. International Journal of Heat and Mass Transfer, 2017, vol. 104, pp.1090-1109. 10.1016/j.ijheatmasstransfer.2016.08.105 . hal-01368480

\section{HAL Id: hal-01368480 \\ https://hal.science/hal-01368480}

Submitted on 19 Sep 2016

HAL is a multi-disciplinary open access archive for the deposit and dissemination of scientific research documents, whether they are published or not. The documents may come from teaching and research institutions in France or abroad, or from public or private research centers.
L'archive ouverte pluridisciplinaire HAL, est destinée au dépôt et à la diffusion de documents scientifiques de niveau recherche, publiés ou non, émanant des établissements d'enseignement et de recherche français ou étrangers, des laboratoires publics ou privés. 


\section{Open Archive TOULOUSE Archive Ouverte (OATAO)}

OATAO is an open access repository that collects the work of Toulouse researchers and makes it freely available over the web where possible.

This is an author-deposited version published in : http://oatao.univ-toulouse.fr/ Eprints ID : 16116

To link to this article : DOI:10.1016/j.ijheatmasstransfer.2016.08.105 URL : http://dx.doi.org/10.1016/j.ijheatmasstransfer.2016.08.105

To cite this version : Rueda Villegas, Lucia and Tanguy, Sébastien and Castanet, Guillaume and Caballina, Ophélie and Lemoine, Fabrice Direct numerical simulation of the impact of a droplet onto a hot surface above the Leidenfrost temperature. (2017) International Journal of Heat and Mass Transfer, vol. 104. pp.1090-1109. ISSN 0017-9310

Any correspondence concerning this service should be sent to the repository administrator: staff-oatao@,listes-diff.inp-toulouse.fr 


\title{
Direct numerical simulation of the impact of a droplet onto a hot surface above the Leidenfrost temperature
}

\author{
Lucia Rueda Villegas ${ }^{\mathrm{b}}$, Sébastien Tanguy ${ }^{\mathrm{a}, *}$, Guillaume Castanet $^{\mathrm{c}, \mathrm{d}}$, Ophélie Caballina ${ }^{\mathrm{c}, \mathrm{d}}$, \\ Fabrice Lemoine ${ }^{\mathrm{c}, \mathrm{d}}$ \\ a University of Toulouse, The Institute of Fluid Mechanics (IMFT), Toulouse, 2, allée du professeur Camille Soula, 31400 Toulouse, France \\ ${ }^{\mathrm{b}}$ University of Toulouse, France \\ ${ }^{\mathrm{c}}$ LEMTA, UMR 7563, Université de Lorraine, France \\ ${ }^{\mathrm{d}}$ LEMTA, UMR 7563, CNRS, France
}

Keywords:

Leidenfrost effect

Phase change

Direct numerical simulation

Evaporation

Boiling

Two-phase flow

Level Set method

Ghost fluid method

\begin{abstract}
A B S T R A C T
In this paper, we compare numerical simulations and experiments on droplets impinging onto a hot surface at a temperature well above the Leidenfrost point, for different impacting Weber numbers ranging from 7 to 45 . We use a novel numerical method for the simulation of two-phase flows with phase change (evaporation and boiling) which accounts for the heterogeneous thermodynamic conditions at the liquid/gas interface. We present the results of experimental and numerical values for the droplet shape, its spreading diameter and its loss of momentum. The numerical simulations determine the timeevolution of the average vapor layer thickness, which is typically from one to two orders of magnitude smaller than the initial droplet diameter. Thermal transfer between the liquid and the gas phases are also investigated both numerically and experimentally. In the numerical results, like in the experiments, the droplet heating increases with the impacting Weber numbers. The fully resolved direct numerical simulations allow for the accurate description of the multi-scale complex problem involving both fluid mechanics and coupled heat and mass transfer.
\end{abstract}

\section{Introduction}

Studying the interaction between droplets and solid surfaces is of great relevance for many industrial and environmental applications, such as wind-driven rain against the facade of buildings, inkjet spray painting, medical sprays inhalation, spray coolers, and fuel injection in combustion engines. Many numerical, theoretical and experimental studies have aimed at understanding the dynamics of drops impinging onto solid and fluid surfaces $[12,69]$. However, the phenomena associated with the drop impingement are far from being fully understood (thermal effects, evaporation, pre-existing and generated waves on the incident fluid surface, etc.). When the solid surface temperature is much higher than the liquid's boiling point, the droplet can levitate upon the hot solid surface. This levitation is induced by the rapid evaporation of the liquid in the neighborhood of the heated solid

\footnotetext{
* Corresponding author.

E-mail addresses: lruedavi@imft.fr (L. Rueda Villegas), tanguy@imft.fr (S. Tanguy), guillaume.castanet@univ-lorraine.fr (G. Castanet), ophelie.caballina@ univ-lorraine.fr (O. Caballina), fabrice.lemoine@univ-lorraine.fr (F. Lemoine).
}

surface. The present work investigates this levitation process, which is called the Leidenfrost effect [42].

Former studies of this phenomenon have been pursued to determine the critical temperature of the hot solid surface, the Leidenfrost point -also denoted Leidenfrost temperature $T_{\text {Leid }}$ - which corresponds to the longest droplet lifetime. The influence of parameters such as the surface roughness $[4,5]$, the Weber number [26], the surface material [5], or the liquid properties [24,25] on the Leidenfrost point have also been evaluated. For instance, a parametric study of $T_{\text {Leid }}$ shows that the surface contamination, the surface material, the liquid subcooling and the liquid degassing have very little influence on the Leidenfrost point [5]. In the present study, the temperature of the surface is far beyond $T_{\text {Leid }}$ to make sure that the droplet levitates upon the surface for the chosen range of Weber numbers. There are three different regimes of interaction between droplets and surfaces: deposition, bouncing, and splashing $[13,26]$. These impact regimes depend on many parameters such as the incident velocity, the surface temperature and the properties of the liquid (viscosity, surface tension ...) but can be determined as a function of the dimensionless temperature $T^{*}$ and the Mundo number $\mathrm{K}[12,13,26]$. In this paper, the focus is on the bouncing regime. 


\begin{tabular}{|c|c|c|c|}
\hline \multicolumn{4}{|c|}{ Nomenclature } \\
\hline$C_{p}$ & isobaric heat capacity $\left(\mathrm{J} \cdot \mathrm{kg}^{-1} \cdot \mathrm{K}^{-1}\right)$ & $T_{\text {wall }}$ & solid surface temperature $(\mathrm{K})$ \\
\hline D & strain tensor $\left(\mathrm{s}^{-1}\right)$ & $\underline{\mathcal{V}}$ & volume $\left(\mathrm{m}^{3}\right)$ \\
\hline$D_{0}$ & initial diameter $(\mathrm{m})$ & $\vec{V}$ & velocity $\left(\mathrm{m} \cdot \mathrm{s}^{-1}\right)$ \\
\hline$D_{m}$ & diffusion coefficient $\left(\mathrm{m}^{2} \cdot \mathrm{s}^{-1}\right)$ & We & Weber number $(-)$ \\
\hline$E_{v}$ & heat taken by the liquid to evaporate $(-)$ & $Y$ & mass fraction $(-)$ \\
\hline$E_{\Delta T}$ & heat entering the droplet $(-)$ & $\beta$ & spreading diameter $(-)$ \\
\hline$\vec{g}$ & gravitational acceleration $\left(\mathrm{m} \cdot \mathrm{s}^{-2}\right)$ & $\varepsilon$ & vapor layer thickness (m) \\
\hline$h_{l g}$ & specific latent heat $\left(\mathrm{J} \cdot \mathrm{kg}^{-1}\right)$ & $\phi$ & level set function $(-)$ \\
\hline$k$ & thermal conductivity $\left(\mathrm{W} \cdot \mathrm{m}^{-1} \cdot \mathrm{K}^{-1}\right)$ & $\kappa$ & interface curvature $\left(\mathrm{m}^{-1}\right)$ \\
\hline$L$ & loss of momentum $(-)$ & $\mu$ & dynamic viscosity $(\mathrm{Pa} \cdot \mathrm{s})$ \\
\hline$L_{r}$ & size of the domain along $\vec{e}_{r}(-)$ & $\rho$ & density $\left(\mathrm{kg} \cdot \mathrm{m}^{-3}\right)$ \\
\hline$L_{z}$ & size of the domain along $\vec{e}_{z}(-)$ & $\sigma$ & surface tension Coefficient $\left(\mathrm{N} \cdot \mathrm{m}^{-1}\right)$ \\
\hline$\dot{m}$ & evaporation rate $\left(\mathrm{kg} \cdot \mathrm{s}^{-1} \cdot \mathrm{m}^{-2}\right)$ & $\nabla$. & divergence operator $(-)$ \\
\hline$M$ & molar mass $\left(\mathrm{kg} \cdot \mathrm{mol}^{-1}\right)$ & $\nabla$ & gradient operator $(-)$ \\
\hline$n$ & number of moles $(-)$ & $\nabla^{t}$ & transpose of the gradient operator $(-)$ \\
\hline$\vec{n}$ & normal vector $(-)$ & {$[.]_{\Gamma}$} & jump condition operator $(-)$ \\
\hline$P$ & pressure $(\mathrm{Pa})$ & $\mathrm{atm}$ & atmospheric condition (subscript) (-) \\
\hline $\mathcal{R}$ & ideal gas constant $\left(\mathrm{J} \cdot \mathrm{mol}^{-1} \cdot \mathrm{K}^{-1}\right)$ & $g$ & gas (subscript) $(-)$ \\
\hline $\operatorname{Re}$ & reynolds number $(-)$ & l & liquid (subscript) $(-)$ \\
\hline$r_{n}$ & velocity ratio $(-)$ & leid & Leidenfrost (subscript) (-) \\
\hline$T$ & temperature $(\mathrm{K})$ & sat & saturation (subscript) (-) \\
\hline$T_{\text {drop }}$ & temperature of the droplet $(\mathrm{K})$ & vap & liquid vapor (subscript) $(-)$ \\
\hline$T_{\text {Leid }}$ & Leidenfrost point $(\mathrm{K})$ & $\Gamma$ & droplet surface (superscript) (-) \\
\hline$T_{\text {sat }}$ & saturation temperature $(\mathrm{K})$ & & \\
\hline
\end{tabular}

In many industrial applications, liquid droplets are initially at the ambient temperature and impact onto the solid surface with an incident velocity. This subcooled regime $\left(T_{\text {drop }}<T_{\text {sat }}\right)$ was investigated in [71] for small spherical sessile droplets $\left(D_{0}<150 \mu \mathrm{m}\right)$. Zhang and Gogos show that the temperature of the interface near the hot surface can reach the liquid boiling point $\left(T_{s a t}\right)$, whereas the temperature in the "outer drop" region remains smaller than $T_{\text {sat }}$. Both evaporation and boiling can then occur at the same time in different regions of the droplet as the liquid inside the drop is heated up. It should be emphasized that the evaporation rate in the outer drop region is found to play a direct role on the heat and mass transfer [24,25,71]. However, in many numerical studies, the droplet is assumed to be already at the saturation temperature before the impingement takes place, which is rather restrictive. Therefore, new numerical approaches are necessary to account for the specific thermodynamic conditions encountered at the liquid interface of a subcooled impinging droplet.

The Leidenfrost effect has thus been widely studied experimentally, but very few numerical simulations exist in the literature. The development of such a numerical model is quite challenging, since hydrodynamic and thermodynamic effects are simultaneously involved at multiple scales. Many previous numerical works deal with boiling flows $[18,22,36,39,55,56,59,60]$, and with evaporation $[28,33,41,45,57,64]$. However, in situations involving heterogeneous thermodynamic conditions at the interface -for instance, a Leidenfrost droplet- the distinction between boiling and evaporation is not always possible and most of those numerical methods are not suitable to simulate this transient regime.

Harvie and Fletcher were among the first to perform axisymmetric simulations of the impact of a droplet onto a hot surface in the Leidenfrost regime $[29,30]$. Their numerical method combines a hydrodynamic model describing the motion of the droplet, and a one-dimensional model simulating the vapor layer's dynamics. In the hydrodynamic model, the Navier Stokes equation is solved using the Continuum Surface Force (CSF) approach [7] that accounts for the capillarity forces, and the Volume Of Fluid (VOF) method that locates the interface. Then, the velocity and the pressure on the vapor layer are determined with a simplified form of the Navier-Stokes equation, which requires knowing the new position of the interface and the vaporization mass rate $\dot{m}$.

Ge and Fan [21] conducted three-dimensional numerical simulations of droplets impacting onto hot surfaces using, like in Harvie and Fletcher [29,30], macroscale and microscale numerical models. At the macroscale, the momentum and mass balance equations are solved using a finite-volume method and a Level Set method to track the interface. The microscale model deals with the flow in the vapor film beneath the droplet. The authors solved the two dimensional momentum and continuity equation with an Arbitrary Lagrangian Eulerian (ALE) technique to compute the velocity and the pressure of the flow in the vapor layer. Finally, a heat transfer model is used to determine the temperature field and the evaporation rate $\dot{m}$. The numerical simulations performed by Ge and Fan were tested with experiments by Groendes and Mesler, Qiao and Chandra, and Wachters and Westerling[27,49,67]. They conclude that their numerical approach is able to predict the dynamic behavior of the droplet from the bouncing to the splashing regimes. The temperature field in the solid surface is also solved and is in good agreement with Groendes and Mesler [27]. The results show that the heat flux is higher in the center of the impact.

Nikolopoulos et al. [47] performed numerical simulations of the evaporation process of $n$-heptane and water droplets impinging onto surfaces below and above the Leidenfrost temperature. The momentum equation is solved using a finite volume approximation coupled with the VOF method and an adaptive local grid refinement technique, to solve accurately the flow in the vapor layer and in the vicinity of the interface. The thermal energy transport is determined using a source term $\dot{Q}\left(\mathrm{Js}^{-1} \cdot \mathrm{m}^{-3}\right)$ that depends on the evaporation rate.

This article investigates the hydrodynamics and the heat transfer during the impact and the levitation of a single droplet onto a hot surface in the bouncing regime. We present numerical 
simulations of small water droplets $\left(D_{0} \sim 130 \mu \mathrm{m}\right)$ initially at ambient temperature $\left(T_{\text {drop }} \sim 25^{\circ} \mathrm{C}\right)$ impinging onto a hot surface with different impinging velocities. The simulations are performed in an axisymmetric coordinate system. Sufficiently weak Weber numbers are considered to ensure that three-dimensional effects can be neglected. The temperature of the solid surface is much higher than the Leidenfrost point $\left(T_{\text {wall }}=800 \mathrm{~K}\right)$ and remains constant all along the simulations. Let us notice that small temperature variations can occur in the solid domain during the droplet spreading. This parameter has a weak influence on the overall phenomenon because the wall superheat is very large in the present simulations and experiments. However, this information on the solid temperature variation is of significant interest for experimental studies, because, from these data, the heat flux in the substrate can be extracted by using inverse methods.

Since the vapor layer thickness is several orders of magnitude thinner than the droplet diameter $[6,8,58]$, our grid is refined near the solid surface in order to perform fully resolved simulations of the interface motion, the phase change, and the heat transfer between the solid, the liquid and the gas phase inside the vapor layer. Section 2 describes the momentum, the continuity, the energy and the mass fraction conservation equations, governing the overall system. The numerical method used to solve these equations is also briefly presented in Section 2. Our method can account for both evaporation and boiling and is suitable to perform the simulation of a subcooled impacting droplet. One difference with respect to the numerical studies previously cited $[21,29,30,47]$, is that the evaporation rate is determined from the temperature field and the mass fraction field using the ClausiusClapeyron law. The experimental set-up is presented in Section 3. Comparisons between the present numerical simulations and the experimental measurements of droplets impinging onto a hot wall with different Weber numbers are reported in Section 4. Finally, the time-evolution of the heat and mass transfer between the surrounding gas and the liquid phase is investigated in Section 5.

\section{Equations and numerical method}

The numerical method used to perform the simulations of the Leidenfrost droplets is thoroughly described in [54]. It enables the resolution of the vapor layer's dynamic, the heat and mass transfers, and the phase change using a strongly refined grid near the solid surface. The stability condition on the time step of explicit algorithms is very restrictive due to the small size of the mesh cells in the vapor layer, especially for the diffusion terms. Therefore, an implicit temporal discretization is used to compute all the diffusion terms in a reasonable computational time. The numerical model is able to handle the heterogeneous thermodynamic conditions at the interface of a subcooled droplet $\left(T_{\text {drop }}<T_{\text {sat }}\right)$ in the Leidenfrost regime (evaporation in the outer drop region, plus evaporation and/or boiling at the bottom). The flow is assumed to be incompressible in order to simplify the simulation. A two-phase flow compressible solver for low Mach number has been developed recently by Huber et al. [34] to perform simulations of the interaction of bubbles with ultrasound waves. However such solvers have not yet been upgraded to deal with phase change. Therefore, an incompressible two-phase flow solver is used in this study. Also, some parameters such as viscosity or density are considered constant in the two phases, although in reality, they can vary with strong thermal gradients, and the mixing of chemical species in the vapor layer.

\subsection{Momentum and mass conservation}

The mass conservation equation for incompressible flows leads to a divergence-free condition on the velocity field, and a jump condition at the liquid/gas interface due to phase change $[22,46,64]:$

$\nabla \cdot \vec{V}=0$

$[\vec{V}]_{\Gamma}=-\dot{m}\left[\frac{1}{\rho}\right]_{\Gamma} \vec{n}$

where $[.]_{\Gamma}=$ is the jump condition operator at the interface defined as follows:

$[A]_{\Gamma}=A_{l}-A_{g}$

The conservation of momentum is satisfied by solving the Navier-Stokes equation for an incompressible flow with a jump condition on the pressure which accounts for the capillary effects, the viscous effects and the phase change:

$$
\begin{aligned}
& \rho\left(\frac{\partial \vec{V}}{\partial t}+\vec{V} \cdot \nabla \vec{V}\right)=-\nabla P+\nabla \cdot(2 \mu \mathbf{D})+\rho \vec{g} \\
& {[P]_{\Gamma}=\sigma \kappa+2\left[\mu \frac{\partial V_{n}}{\partial n}\right]_{\Gamma}-\dot{m}^{2}\left[\frac{1}{\rho}\right]_{\Gamma}}
\end{aligned}
$$

where $\partial V_{n} / \partial n=\nabla \vec{V} \cdot \vec{n}$ is the derivative in the normal direction at the interface. The position and the evolution of the interface are computed using the Level Set Method [62]. To determine the velocity and the pressure from Eqs. (1) and (3), the projection method from [64] is used with the following modifications: (i) the viscous terms must be computed using an implicit discretization to handle the viscosity jump, (ii) and the numerical method must account for the additional jump conditions due to phase change reported in Eqs. (2) and (4).

To compute an implicit discretization of viscous terms for the simulation of phase change, we use the Ghost Fluid SemiConservative viscous Method (GFSCM) developed by Lalanne et al. [40]. In the framework of Level Set/Ghost fluid methods, two main numerical discretizations are commonly used. They are developed by Kang et al. [37], and Sussman et al. [63] to compute the viscous terms for the simulation of incompressible two-phase flows when the viscosity is discontinuous across the interface [40]. In [37], the divergence of the viscous stress tensor is contracted in a Laplacian operator of the velocity component $\mu \Delta \vec{V}$. The other term, the jump of the normal viscous stresses at the interface resulting from the jump of viscosity, is added explicitly to the pressure jump condition while solving the Poisson equation for the pressure. This method is referred to in [40] as the Ghost Fluid Primitive viscous Method (GFPM). In [63], inspired by a previous work of Liu et al. [44], the divergence of the viscous stress tensor is discretized in a similar way to CSF methods and the contribution of the normal viscous stresses is not added to the pressure jump condition at the interface. In [40], this method is referred to as the Ghost Fluid Conservative viscous Method (GFCM).

While the implicit discretization of viscous terms can be done using the GFPM algorithm, the method cannot be applied to simulations with phase change because the computation of the normal viscous stresses is not possible if the velocity field is not continuous at the interface. The GFCM is perfectly suitable for the simulation of phase change. However, the implementation of an implicit method leads to one complex coupled parabolic linear system for all the velocity components. With our method, the GFSCM, if the viscosity is considered as a constant, the divergence of the viscous stress tensor is split into two terms. The first, $\nabla \cdot(\mu \nabla \vec{V})$, is used to compute the intermediate velocity field which does not respect the divergence-free condition (predictor step of the projection method). The second-order cross-derivatives, $\nabla \cdot\left(\mu \nabla^{t} \vec{V}\right)$, are non-zero only at the interface and are included in the pressure 
jump condition when the Poisson equation is solved. Accounting for the variations of the viscosity would imply to solve a complex linear system coupling all the velocity components. Such a formulation has been proposed recently [43] for a piecewise constant viscosity but a further work would be required to use this formulation with a jump condition on the velocity due to phase change.

The algorithm from Nguyen et al. [46] solves the Navier Stokes equation with appropriate jump conditions accounting for a reactive interface between two incompressible immiscible fluids with different densities. In the present work, an algorithm inspired by [46] and developed in [64] is implemented to compute a velocity field extension which respects a divergence-free condition $[54,64,65]$ more suitable for the simulation of droplet evaporation.

\subsection{Energy and mass fraction conservation}

The energy conservation is expressed with the following equation and jump condition on the thermal flux at the interface which allows accounting for phase change:

$\rho C_{p}\left(\frac{\partial T}{\partial t}+\vec{V} \cdot \nabla T\right)=\nabla \cdot(k \nabla T)$

$[k \nabla T \cdot \vec{n}]_{\Gamma}=\dot{m}\left(h_{\text {lg }}+\left(C_{p_{l}}-C_{p_{\text {vap }}}\right)\left(T_{\text {sat }}-T^{\Gamma}\right)\right)$

$[T]_{\Gamma}=0$

The energy conservation equation is solved using the numerical method from Gibou et al. [22]. In this algorithm, the temperature field is first computed in the gas phase and then in the liquid phase with a Dirichlet condition $T^{\Gamma}$ at the liquid/gas interface. The temperature field of each phase is extended afterwards using the extrapolation technique in [3] and the evaporation rate $\dot{m}$ is deduced from Eq. (6). In [22], the authors assume that the temperature at the interface is constant and equal to $T^{\Gamma}=T_{\text {sat }}$ because they consider the boiling of a single-species system. However, it was pointed out previously that for Leidenfrost droplets in the subcooled regime, the thermodynamic condition at the interface is heterogeneous since the temperature varies depending on the position. To determine $T^{\Gamma}$, the thermodynamic local equilibrium conditions for a pure liquid in contact with a gas mixture should be invoked. By assuming that the gas mixture behaves as an ideal gas, the total pressure can be defined as the sum of each individual component

$P_{\text {atm }}=P_{\text {vap }}+P_{g}$

$P_{\text {vap }}$ and $P_{g}$ are determined as follow:

$P_{\text {vap }} \mathcal{V}=n_{\text {vap }} \mathcal{R} T$

$P_{g} \mathcal{V}=n_{g} \mathcal{R} T$

The mass fraction of vapor is defined by the following relation:

$Y_{\text {vap }}=\frac{n_{\text {vap }} M_{\text {vap }}}{n_{\text {vap }} M_{\text {vap }}+n_{g} M_{g}}$

By replacing $n_{\text {vap }}$ and $n_{g}$ in Eq. (11) using Eqs. (9) and (10), the mass fraction of vapor can be rewritten as follows:

$Y_{\text {vap }}=\frac{P_{\text {vap }} M_{\text {vap }}}{P_{\text {vap }} M_{\text {vap }}+\left(P_{\text {atm }}-P_{\text {vap }}\right) M_{g}}$

At the interface, the saturated vapor pressure of the liquid component is determined by the Clausius-Clapeyron law:

$P_{\text {vap }}^{\Gamma}=P_{a t m} e^{\frac{h_{l g} M_{\text {vap }}}{R}\left(\frac{1}{T^{T}}-\frac{1}{T_{\text {sat }}}\right)}$
Therefore, by rewritting Eqs. (12) and (13), the non-uniform temperature at the interface $T^{\Gamma}$ can be defined as follows:

$$
\begin{gathered}
P_{\text {vap }}^{\Gamma}=\frac{Y_{\text {vap }}^{\Gamma} P_{a t m} M_{g}}{M_{\text {vap }}-\left(M_{\text {vap }}-M_{g}\right) Y_{\text {vap }}^{\Gamma}} \\
T^{\Gamma}=\frac{h_{\text {lg }} M_{\text {vap }} T_{\text {sat }}}{h_{\text {lg }} M_{\text {vap }}-\mathcal{R} T_{\text {sat }} \ln \left(\frac{P_{\text {vap }}^{\Gamma}}{P_{\text {atm }}}\right)}
\end{gathered}
$$

Knowing the vapor concentration at the interface $\left(Y_{\text {vap }}^{\Gamma}\right)$, the temperature $T^{\Gamma}$ can be determined by using the Eq. (15). The vapor concentration is computed by solving Eq. (16) with the jump condition (17) due to the conservation of the chemical species vap:

$$
\begin{aligned}
& \rho\left(\frac{\partial Y_{\text {vap }}}{\partial t}+\vec{V} \cdot \nabla Y_{\text {vap }}\right)=\nabla \cdot\left(\rho D_{m} \nabla Y_{\text {vap }}\right) \\
& {\left[\rho D_{m} \nabla Y_{\text {vap }} \cdot \vec{n}\right]_{\Gamma}=-\dot{m}\left[Y_{\text {vap }}\right]_{\Gamma}}
\end{aligned}
$$

By remarking that for a single component liquid the jump condition can be simplified, we obtain the following Robin boundary condition at the interface for the mass fraction field:

$\dot{m} Y_{\text {vap }}^{\Gamma}+\left.\rho_{g} D_{m} \nabla Y_{\text {vap }} \cdot \vec{n}\right|^{\Gamma}=\dot{m}$

Therefore, Eq. (16) and the boundary condition at the interface Eq. (18) (Robin condition), are solved with the technique presented in [48] which is of second order in space and maintains a symmetric definite linear system.

\subsection{Interface advection with a Level Set method}

The Level Set method is used to determine the position of the liquid gas interface for each time step [19,22,46,48,62,64]. A level set function $\phi$ is introduced so that the isocontour of $\phi=0$ represents the interface. The temporal evolution of $\phi$ is computed by solving the following convection equation:

$\frac{\partial \phi}{\partial t}+\vec{V}_{\text {int }} \cdot \nabla \phi=0$

The function $\phi$ represents the distance to the interface (by definition $\phi>0$ in the liquid, $\phi<0$ in the gas). The interface velocity, $\vec{V}_{i n t}$, is given by

$\vec{V}_{\text {int }}=\vec{V}_{l}+\frac{\dot{m}}{\rho_{l}} \vec{n}=\vec{V}_{g}+\frac{\dot{m}}{\rho_{g}} \vec{n}$

where $\vec{n}$ is the normal vector pointing towards the interior of the liquid phase. A reinitialization step is then performed to ensure that the level set function remains a signed distance in the computational domain. This can be achieved by solving the following Partial Differential Equation (PDE) at the end of a time step [62]:

$\frac{\partial d}{\partial \tau}=\operatorname{sign}(\phi)(1-|\nabla d|)$

where $d$ is the reinitialized distance, $\tau$ a fictitious time and $\operatorname{sign}(\phi)$ the smoothed signed function defined in [62]. Let us notice that two temporal iterations are required at every time step to maintain $d$ as a signed distance. Finally, the level set function allows to determine the normal vector and the curvature $\kappa$ at the interface:

$\vec{n}=\frac{\nabla \phi}{|\nabla \phi|}$

$\kappa(\phi)=-\nabla \cdot \vec{n}$ 
Table 1

Fluid properties (units SI) for the simulation presented in Section 2.4.

\begin{tabular}{|c|c|c|c|c|c|c|c|c|c|}
\hline Fluids & $\rho$ & $\mu$ & $C_{p}$ & $\kappa$ & $\sigma$ & $T_{\text {sat }}$ & $h_{l g}$ & $D_{m}$ & $M$ \\
\hline Acetone (liquid) & 700 & $3.2610^{-4}$ & 2000 & 0.161 & 0.0237 & 329 & 518,000 & $5.210^{-5}$ & $5.810^{-2}$ \\
\hline Gas mixing & 1 & $10^{-5}$ & 1000 & 0.052 & - & - & - & - & $2.910^{-2}$ \\
\hline
\end{tabular}

Table 2

Parameters of the simulation presented in Section 2.4 .

\begin{tabular}{|c|c|c|c|c|c|c|}
\hline & $T_{\infty}$ & $Y_{\infty}$ & $D_{0}$ & $V_{z}$ & $L_{r}$ & $L_{z}$ \\
\hline$R e=20$ & $700 \mathrm{~K}$ & 0 & $200 \mu \mathrm{m}$ & $-2 \mathrm{~m} \cdot \mathrm{s}^{-1}$ & $4 D_{0}$ & $8 D_{0}$ \\
\hline
\end{tabular}

$300 \quad 325 \quad 350 \quad 375 \quad 400 \quad 425 \quad 450 \quad 475 \quad 500 \quad 525 \quad 550 \quad 575 \quad 600$

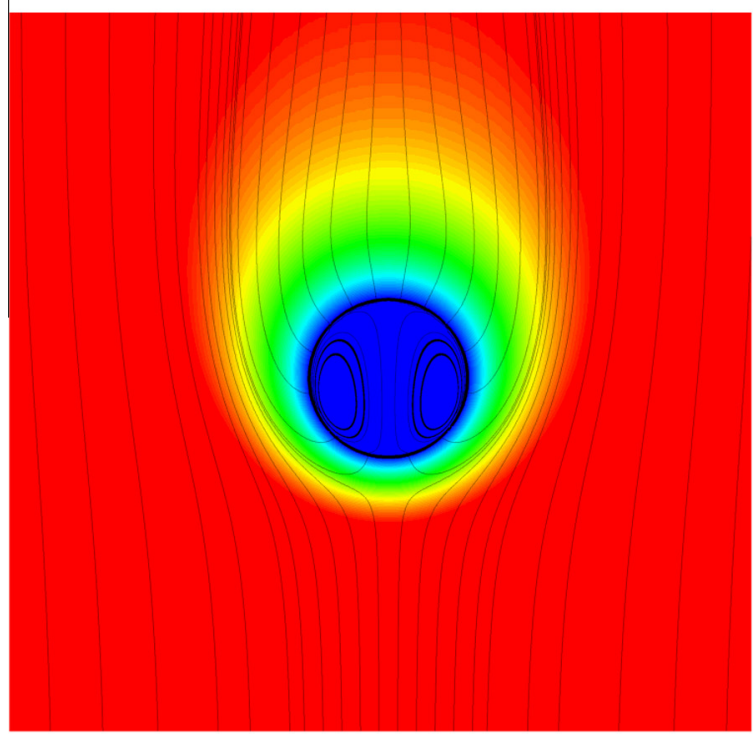

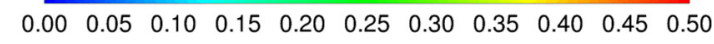

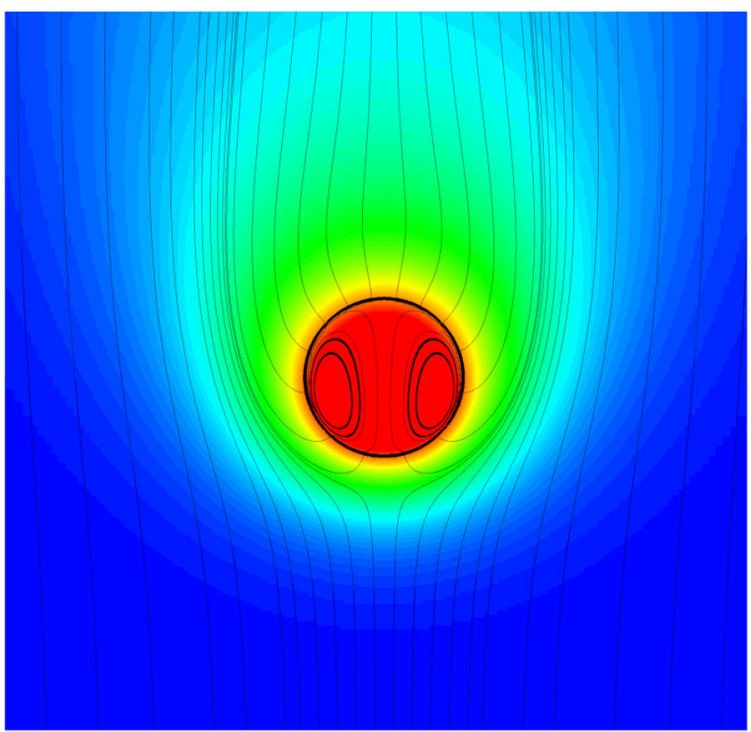

Fig. 1. Temperature field (left), mass fraction field (right), streamlines and interface position around a moving droplet which evaporates in a hot gas.
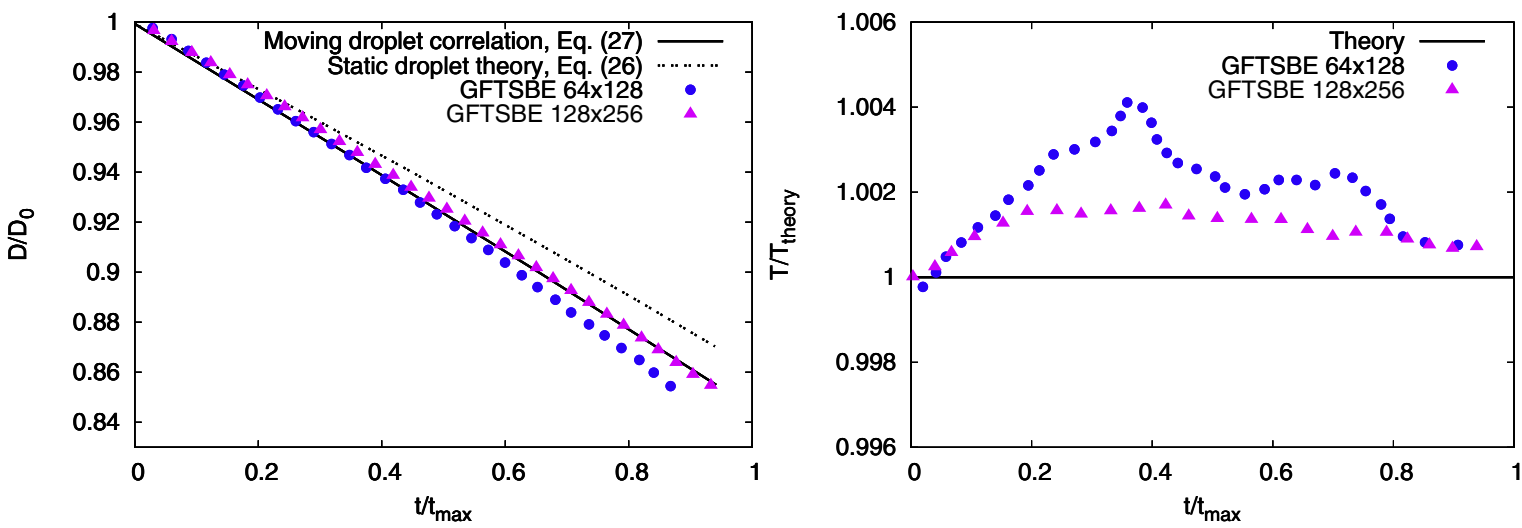

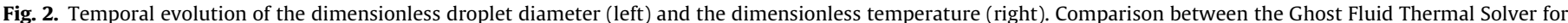
Boiling and Evaporation (GFTSBE) method and a semi-empirical correlation.

\subsection{Direct numerical simulation of the evaporation of a moving droplet}

This section reports a numerical benchmark to assess the relevance and accuracy of the present numerical method. The results from the simulation of the evaporation of a moving droplet are compared with the correlation of Renksizbulut et al. which is valid under the following conditions [50]:

- The droplet remains spherical (Weber number sufficiently small).

- The flow field is axisymmetric and laminar. 


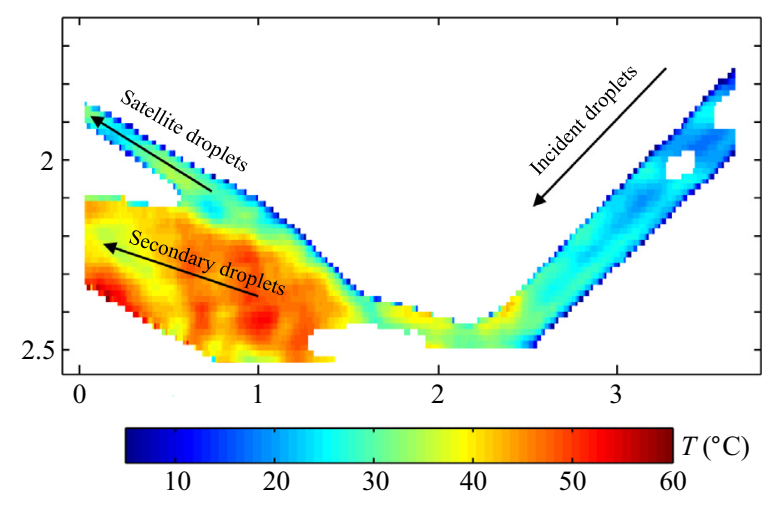

Fig. 3. An example of temperature distribution obtained by 2cPLIF thermometry in the case of a rebound with satellite droplets $\left(D_{0}=133 \mu \mathrm{m}, \vec{V}_{0}=4.3 \mathrm{~m} / \mathrm{s}\right.$, $\left.T_{\text {wall }}=670^{\circ} \mathrm{C}, W e=35\right)$. Figure adapted from "Energy balance of droplets impinging onto a wall heated above the Leidenfrost temperature by P. Dunand, G. Castanet M. Gradeck, D. Maillet and F. Lemoine, International Journal of Heat and Fluid Flow, Vol. 44 (2013) [16]". Adapted with permission.

- The gravity, the thermal radiation, the Soret and Dufour effects and the viscous dissipation are negligible.

- The gas/liquid interface is in thermodynamic equilibrium.

- The gas is insoluble in the liquid phase.

The Renksizbulut et al. correlation is based on the evaporation of a static droplet theory developed by Spalding in [61]. The static droplet theory allows to determine the global mass flow rate $\dot{M}(t)$ as follows:

$\dot{M}(t)=\pi D_{\Gamma}(t) \rho_{g} D_{m} \operatorname{Sh} B_{M}$

where the mass transfer coefficient $B_{M}$ and the Sherwood number Sh are defined as follows:

$B_{M}=\frac{Y^{\Gamma}-Y_{\infty}}{1-Y^{\Gamma}}$

$S h=2 \frac{\ln \left(1+B_{M}\right)}{B_{M}}$

The temporal evolution of the droplet diameter $D_{\Gamma}(t)$ is determined by integrating Eq. (24):

$D_{\Gamma}(t)=\sqrt{D_{0}^{2}-\frac{4 \rho_{g} D_{m}}{\rho_{l}} \ln \left(1+B_{M}\right) t}$

The static theory cannot be directly applied to a falling droplet since the motion of the liquid has an influence on the heat and mass transfer. Renksizbulut et al. modify the Sherwood number with the Reynolds number $(R e)$ and the Schmidt number $(S c)$ in order to account for the coupling between the fluid motion and the forced convection at the droplet surface.

$\frac{S h}{\left(1+B_{M}\right)^{0.7}}=2+0.87 R e^{\frac{1}{2}} S c^{\frac{1}{3}}$

Sc is given by

$S c=\frac{\mu_{g}}{\rho_{g} D_{m}}$

This correlation for the Sherwood number allows to determine the mass flow rate by using Eq. (24). Then, the temporal evolution of the droplet diameter can be deduced by integrating Eq. (24) using Eq. (27) to determine the Sherwood number.

The numerical simulations are performed in an axisymmetric coordinate system with a moving frame to maintain the droplet in the center of the computational domain. Indeed, the evaporation rate being very low, the droplet covers an important distance before a significant evaporation occurs. The fluids properties and the parameters of the numerical simulation are reported in Tables 1 and 2 . The computational domain dimensions are defined to minimize the containment effects. The equations are computed with two computational grids containing $64 \times 128$ and $128 \times 256$ (with respectively 16 and 32 grid cells to discretize a droplet radius). For both meshes, the grid is uniform in the neighborhood of the droplet (until a distance of $D_{0} / 2$ from the interface) and a nonuniform grid is used outside this region to minimize the number of grid cells. The gravity is neglected and a constant relative velocity $V_{z}$ is imposed between the droplet and the gas phase. The temperature and the mass fraction fields are initialized with the theoretical solution of the motionless case [61]. The energy and species conservation equations are solved with Dirichlet boundary conditions at the bottom edge of the domain $\left(T_{\infty}\right.$ and $\left.Y_{\infty}\right)$ and Neumann boundary conditions everywhere else. For the NavierStokes equation, we use the following boundary conditions:

- Inflow condition at the bottom to ensure the constant relative velocity.

- Slip condition at the right side.

- Outflow boundary condition at the top.

Comparison tests between the Renksizbulut et al. correlation and the direct numerical simulations have been conducted considering a $200 \mu \mathrm{m}$ droplet of acetone placed in a hot gas mixture at $700 \mathrm{~K}$ with an initial relative velocity of $2 \mathrm{~m} / \mathrm{s}$. The temperature field and the mass fraction field are displayed in Fig. 1. This figure shows the streamlines inside and outside the droplet to highlight the coupling between the external flow and the Stefan flow. The temporal evolutions of the droplet diameter (until it decreases of $15 \%$ ) and the droplet temperature are plotted in Fig. 2. The latter shows a very good agreement between the numerical results and the theoretical evolution based on (27). The figure also brings out the convergence of the numerical method. Furthermore, it shows that the numerical results using the coarse grid are close to the theoretical solution.

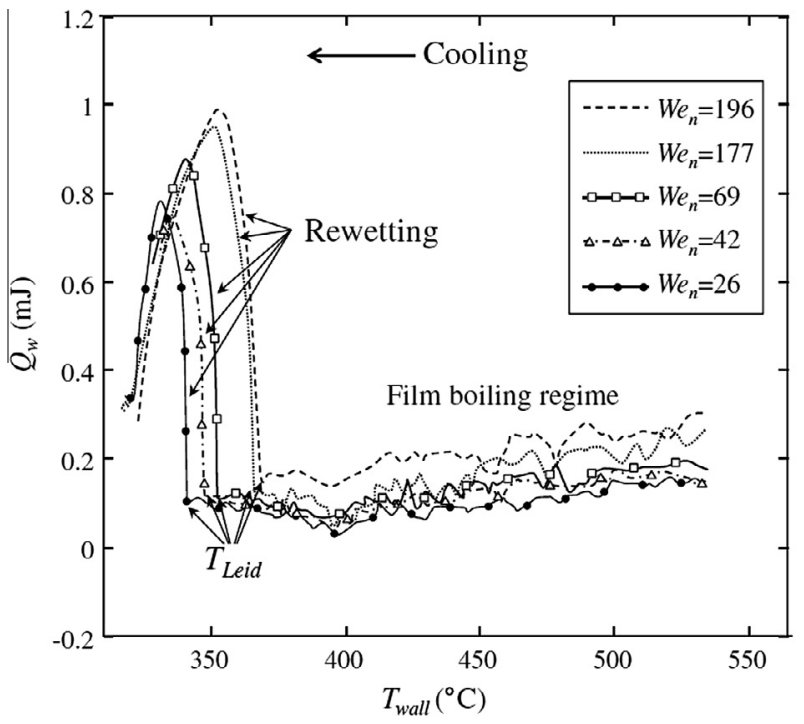

Fig. 4. Typical evolution of the heat removed per droplet as a function of the wall temperature obtained by the inverse heat conduction model presented by Dunand et al. $[14,15]$. Droplet size and injection frequency are fixed in the measurements $(D=180 \mu \mathrm{m}$ and $f=12 \mathrm{kHz})$ 
Table 3

Fluid properties (units SI) for the simulations presented in Sections 4 and 5.

\begin{tabular}{|c|c|c|c|c|c|c|c|c|c|}
\hline Fluids & $\rho$ & $\mu$ & $C_{p}$ & $\kappa$ & $\sigma$ & $T_{\text {sat }}$ & $h_{\lg }$ & $D_{m}$ & $M$ \\
\hline Water (liquid) & 1000 & 0.00113 & 4180 & 0.6 & 0.07 & 373 & $2,300,000$ & $2.010^{-5}$ & $1.810^{-2}$ \\
\hline Air (gas) & 1.226 & 0.00003 & 1000 & 0.046 & - & - & - & - & $2.89710^{-2}$ \\
\hline
\end{tabular}

Table 4

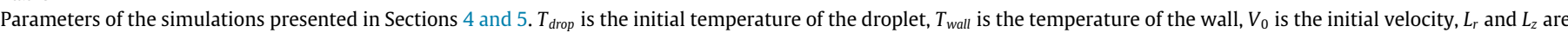
the size of the domain along $\vec{e}_{r}$ and $\vec{e}_{z}$, Grid is the number of grid cells and $D_{0}$ is the initial diameter of the droplet.

\begin{tabular}{|c|c|c|c|c|c|c|c|}
\hline Case & $T_{\text {drop }}$ & $T_{\text {wall }}$ & $D_{0}$ & $V_{0}$ & Grid & $L_{r}$ & $L_{z}$ \\
\hline$W e=7.4$ & $290 \mathrm{~K}$ & $800 \mathrm{~K}$ & $130 \mu \mathrm{m}$ & $2 \mathrm{~m} \cdot \mathrm{s}^{-1}$ & $96 \times 192$ & $2 D_{0}$ & $1.5 l_{r}$ \\
\hline$W e=11$ & $290 \mathrm{~K}$ & $900 \mathrm{~K}$ & $130 \mu \mathrm{m}$ & $2.43 \mathrm{~m} \cdot \mathrm{s}^{-1}$ & $96 \times 192$ & $2 D_{0}$ & $1.5 l_{r}$ \\
\hline$W e=15$ & $290 \mathrm{~K}$ & $900 \mathrm{~K}$ & $130 \mu \mathrm{m}$ & $2.842 \mathrm{~m} \cdot \mathrm{s}^{-1}$ & $96 \times 192$ & $2 D_{0}$ & $1.5 l_{r}$ \\
\hline$W e=20$ & $290 \mathrm{~K}$ & $900 \mathrm{~K}$ & $130 \mu \mathrm{m}$ & $3.28 \mathrm{~m} \cdot \mathrm{s}^{-1}$ & $96 \times 192$ & $2 D_{0}$ & $1.5 l_{r}$ \\
\hline$W e=25.2$ & $300 \mathrm{~K}$ & $746 \mathrm{~K}$ & $133 \mu \mathrm{m}$ & $3.7 \mathrm{~m} \cdot \mathrm{s}^{-1}$ & $96 \times 384$ & $2 D_{0}$ & $3 l_{r}$ \\
\hline$W e=44.8$ & $290 \mathrm{~K}$ & $814 \mathrm{~K}$ & $120 \mu \mathrm{m}$ & $5.18 \mathrm{~m} \cdot \mathrm{s}^{-1}$ & $96 \times 384$ & $2 D_{0}$ & $3 l_{r}$ \\
\hline
\end{tabular}
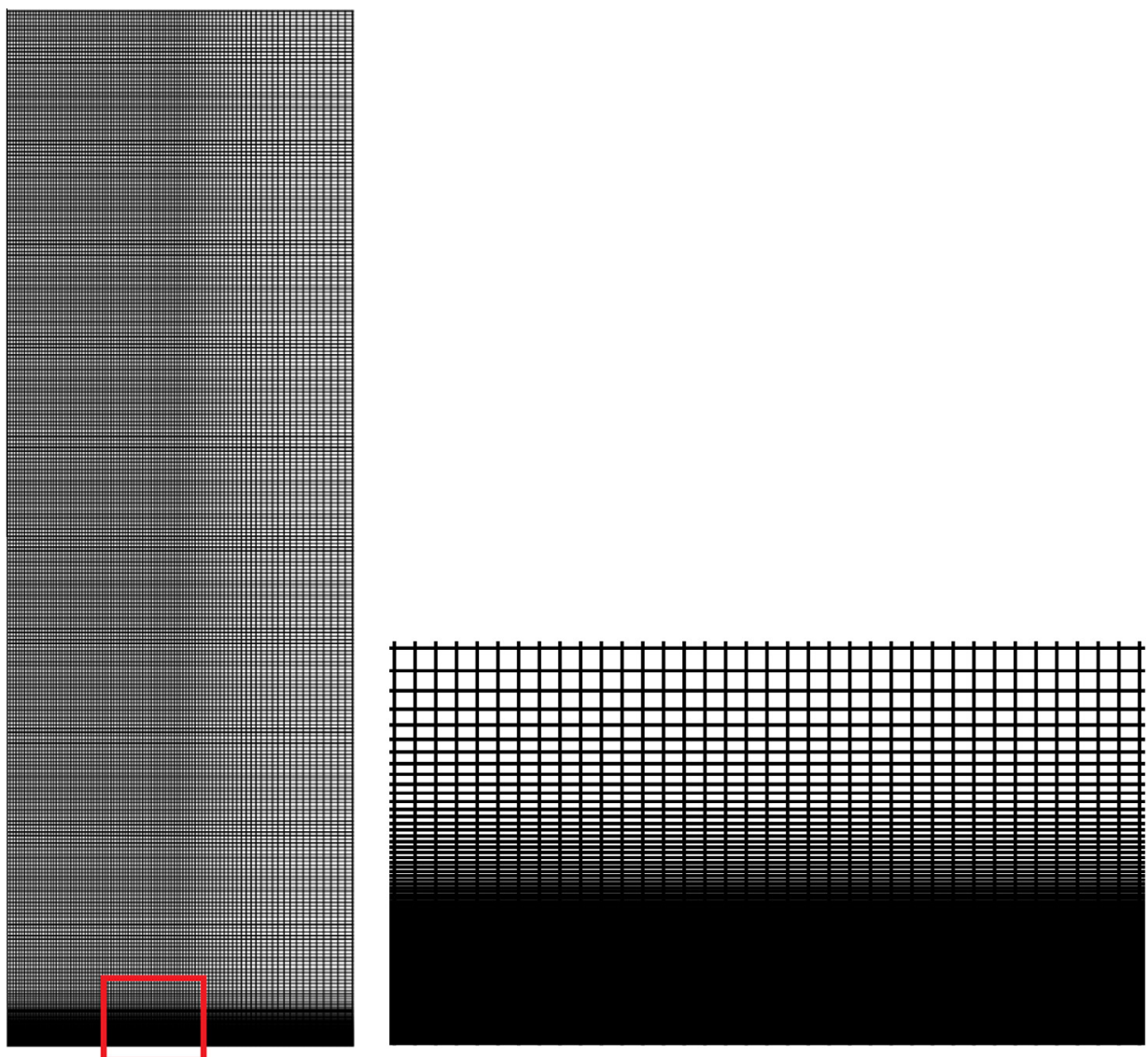

Fig. 5. Strongly refined computational grid ( $96 \times 384$ meshes) for the simulations presented in Sections 4 and 5 with $W e=25.2$ and $W e=44.8$.

\section{Table 5}

Comparison between the numerical simulations and the present experiments $[11,16]$ for the Leidenfrost water droplet with $W e=7.4$.

\begin{tabular}{ccc}
\hline & $\begin{array}{c}\text { Spreading diameter } \\
\beta_{\max }\end{array}$ & $\begin{array}{c}\text { Restitution } \\
\text { coefficient } r_{n}\end{array}$ \\
\hline $\begin{array}{c}\text { Experimental } \\
\text { measurements }\end{array}$ & 1.4 & 0.59 \\
$\begin{array}{c}\text { Numerical simulation } \\
96 \times 192\end{array}$ & 1.57 & 0.61 \\
Numerical simulation \\
$144 \times 288$
\end{tabular}

\section{Experimental setup}

Numerical simulations are compared with experimental results. This section only presents the main outlines of these experimental methods. A more detailed description can be found in [16,17]. A piezoelectric droplet generator is used to produce mono-size and equally-spaced droplets at a relatively high frequency (on the order of 10,000 droplets per second). The droplet generator is rotated in order to adjust the angle of impact. Also, the inlet pressure and the diameter of the injector orifice can be modified to change the size and the velocity of the droplets. In the present 


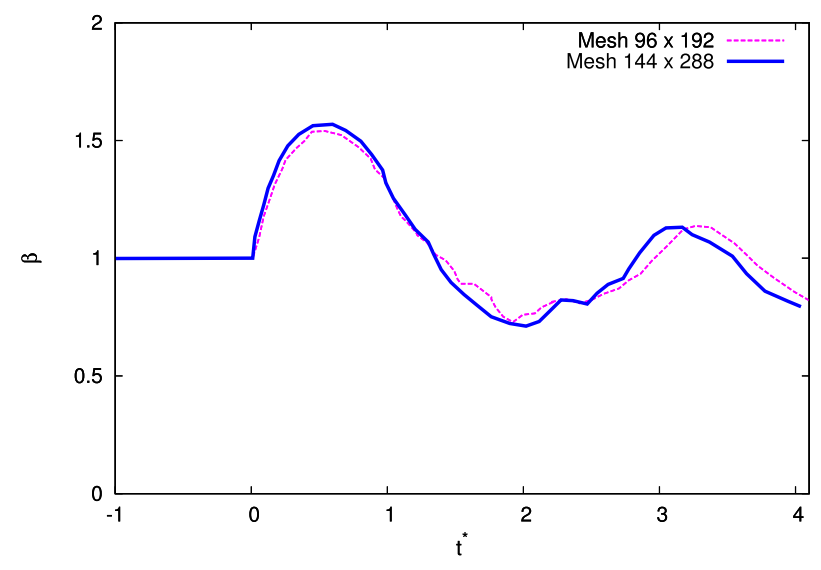

Fig. 6. Temporal evolution of the dimensionless spreading diameter with $W e=7.4$

study, the initial diameter of the droplets varies from $80 \mu \mathrm{m}$ to $250 \mu \mathrm{m}$, the velocity is set to a few $\mathrm{m} / \mathrm{s}$ while the temperature of the injector is regulated at $25^{\circ} \mathrm{C}$. The droplets impinge a thin disk of nickel heated by induction. The upper surface of the nickel target is polished so as to obtain an averaged rugosity $R a$ below $1 \mu \mathrm{m}$. Several measurement techniques are implemented:

- High-speed shadowgraphy allows visualizing the droplet motion and deformation. Given the extreme brevity of the deformation process (typically $100 \mu \mathrm{s}$ ), time-resolved observations imply acquisition rates close to 100,000 frame-per second.
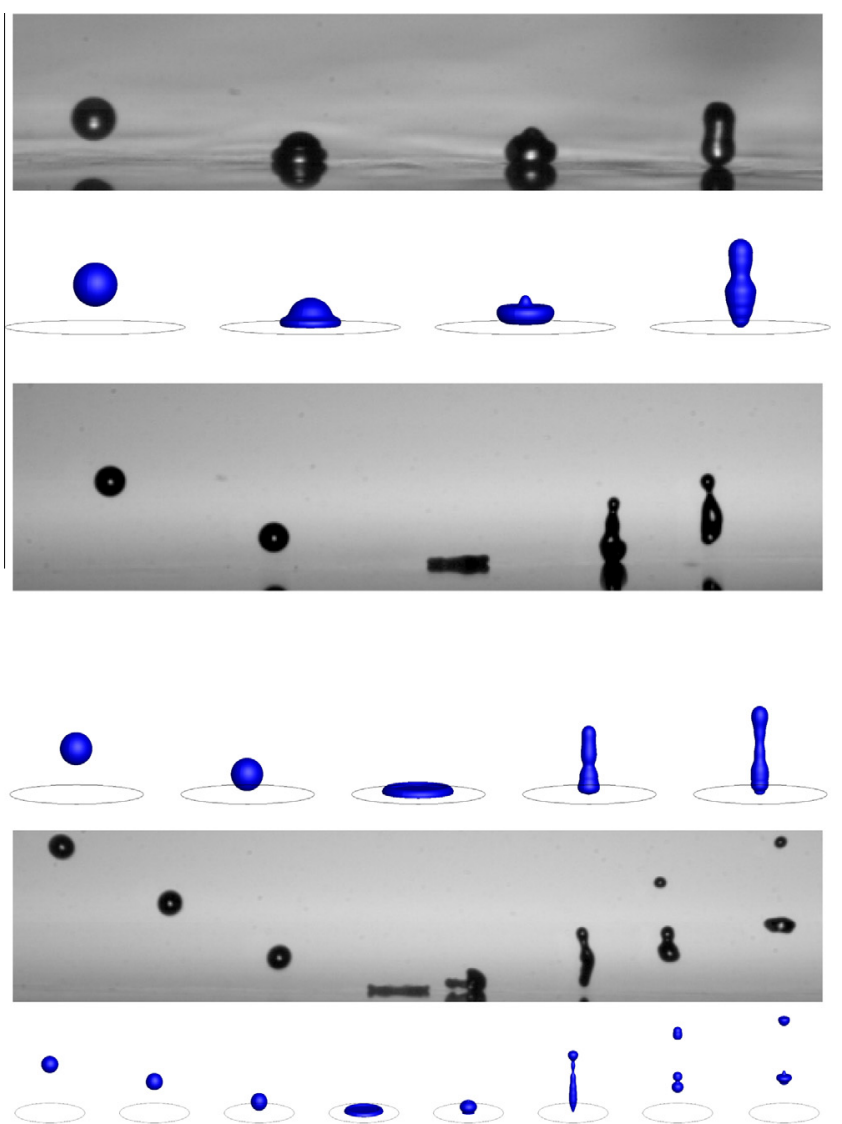

Fig. 7. Experimental images and 3D visualization of an axisymmetric numerical simulation of the impact of a droplet onto a hot surface at $W e=7.4, W e=25.2$ and $W e=44.8$ in the Leidenfrost regime.
Important parameters such as the incident angle, the normal and tangential velocities of ongoing and outcoming droplets, the residence time, the spreading diameter are deduced from the image processing [9].

- The temperature evolution of the droplets is measured using the two-color Planar Laser-Induced Fluorescence (2cPLIF) thermometry [15-17]. The liquid is seeded with a temperaturedependent fluorescent dye. The fluorescence of the droplets is induced by a laser sheet and observed by means of two cameras equipped with distinct bandpass filters. The image ratio of the two cameras is converted into a temperature after a calibration of the measurement system. Because the exposure time of the cameras is much longer than the duration of the deformation process, only temporally averaged temperature can be obtained as illustrated in Fig. 3.

- An infrared camera provides the temperature at the rear face of the thin nickel target. This temperature is monitored during the cooling of the nickel target once the heating by the induction is switched off. A specific inverse heat conduction model has been developed to determine the heat removed by the droplets from the temperature evolution [16]. As an example, Fig. 4 shows the evolution of Qw during a cooling sequence as a function of the wall temperature $T_{\text {wall }}$. The cooling starts in the film boiling regime (right hand side of Fig. 4) and finishes in the nucleate boiling regime (left hand side of Fig. 4). When the wall reaches the Leidenfrost temperature, liquid starts rewetting the wall, which induces a sharp increase in the heat removal. The rewetting "overshoot" observed in Fig. 4 is the consequence of the increase of the dynamic Leidenfrost temperature with the Weber number. Due to this increase, the rewetting takes place at a higher wall temperature for the high Weber numbers. At the same time, the heat flux is an increasing function of the wall temperature in the nucleate boiling. Once the wall is wetted, the Weber number has no effect on the heat flux. The heat flux becomes an increasing function of the wall temperature and the inlet liquid flow rate exclusively.

Finally, energy conservation can be invoked to estimate indirectly the heat associated to evaporation, as the heat removed from the wall by the droplets and the sensible heat gained by the droplets are known from the previous measurements techniques. The respective contributions of the liquid sensible heat, the heat

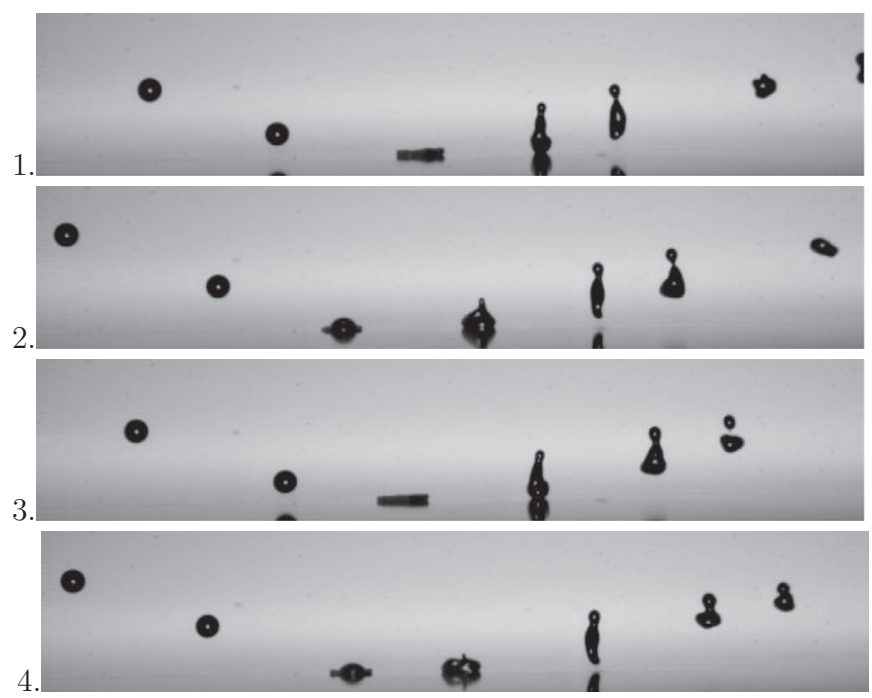

Fig. 8. Experimental images of droplets bouncing onto a hot surface at $W e=25.2$. 
(a)

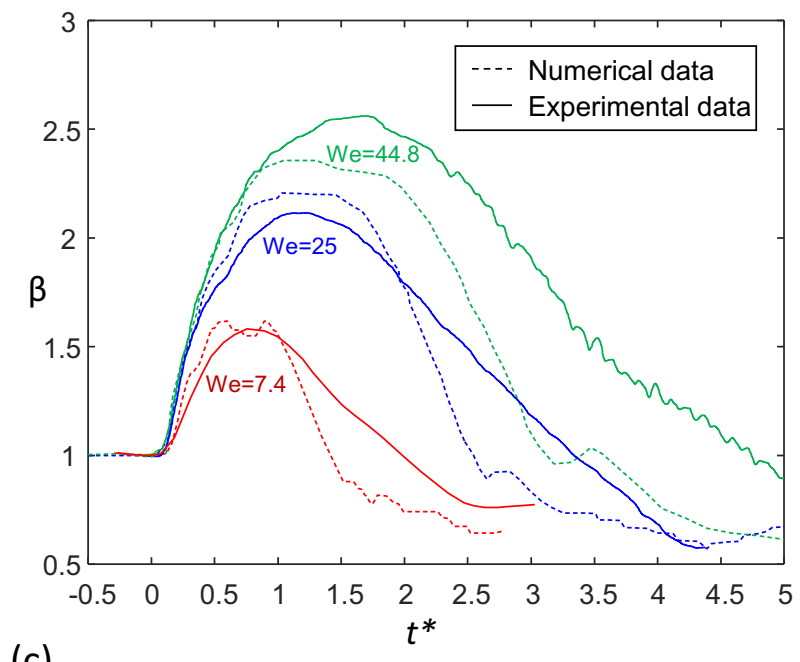

(c)

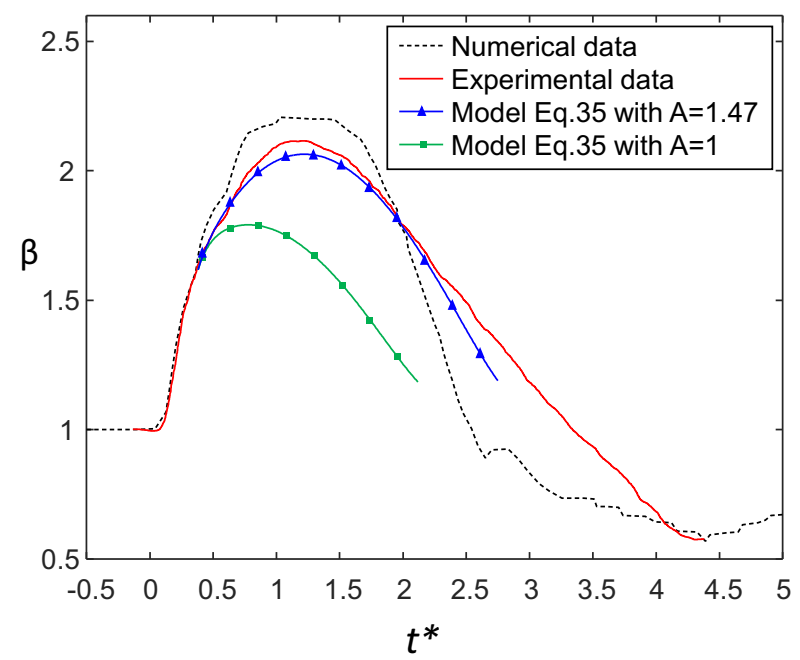

(b)

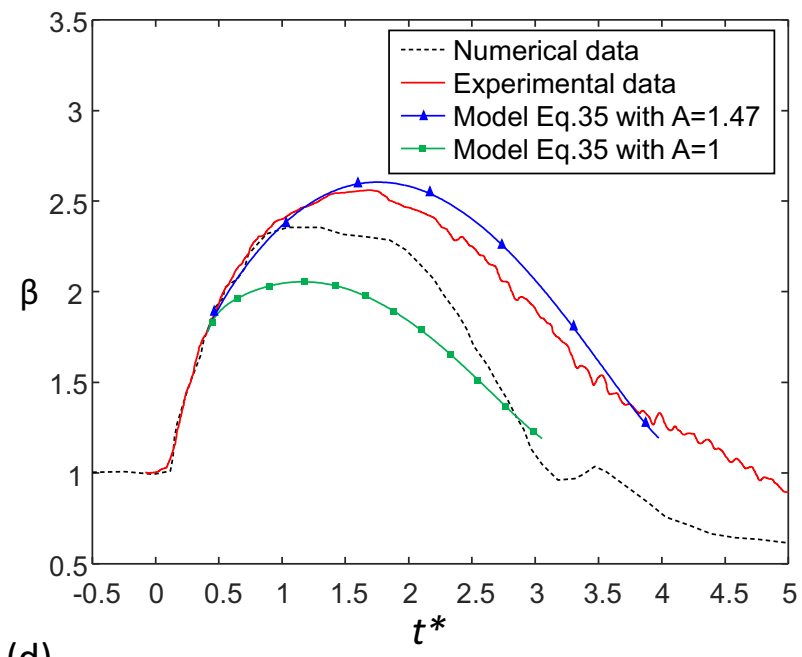

(d)

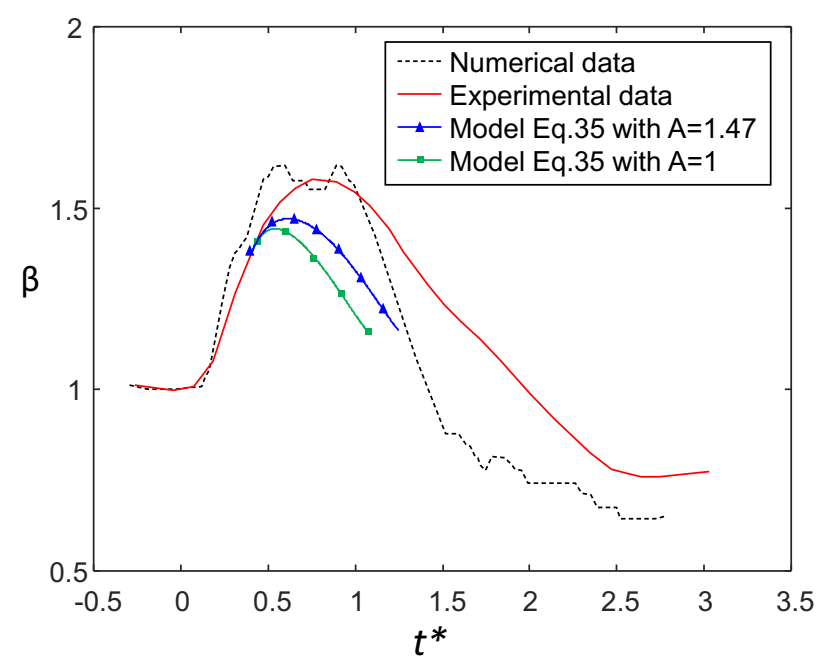

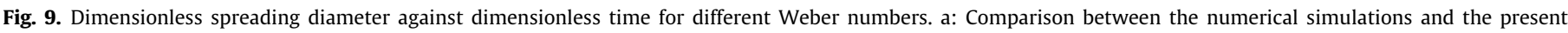
experiments, b-d: Comparison to the model of Eq. (35) with $A=1$ and $A=1.47$ for $W e=44.8$ (b), $W e=25.2$ (c) and $W e=7.4$ (d).

of evaporation, and the heat removed from the wall are presented in [16].

\section{Drop impact dynamics}

Axisymmetric numerical simulations are performed for different impinging velocities. The corresponding Weber numbers based on the droplet velocity projected in the normal direction to the wall are $7.4,11,15,20,25.2$, and 44.8:

$W e=\frac{\rho_{l} D_{0}\left(\vec{V}_{\text {impact }} \cdot \vec{e}_{z}\right)^{2}}{\sigma}$

For these moderate Weber numbers covering the bouncing regime, the experimental results show that the droplet shape remains approximatively axisymmetric. The evolution of the droplet shape, its spreading diameter, its bouncing velocity, and the formation of secondary droplets is discussed in the following sections. Since some physical properties can vary for strong thermal gradients, average and constant values have been considered in our simulations. These averaged properties are reported in Table 3. The parameters of the numerical simulations are given in Table 4.
The vapor layer beneath the droplet being very thin, small grid cells are required to accurately solve the vapor flow in this singular region. Therefore, the numerical simulations are performed on a non-uniform structured grid which is strongly refined near the surface as it is shown in Fig. 5. To preserve the accuracy of the spatial discretization, the grid has a smooth continuous transition between large and small cells.

\subsection{Direct numerical simulation of a water droplet impinging onto a hot surface with We $=7.4$}

The numerical simulation of a droplet impacting onto a solid surface above the Leidenfrost point is quite challenging. Indeed, since the vapor layer is several orders of magnitude smaller than the droplet diameter, a locally refined grid is necessary to capture its formation. To obtain the numerical results within a reasonable time, an implicit temporal discretization must be applied to compute the diffusion terms (viscous terms, thermal conduction, mass fraction diffusion) [40]. Also, the extrapolation techniques which are used to solve the hyperbolic partial differential equations are implemented using an implicit discretization in the refined direction. This ensures that the extrapolation will be done in a 


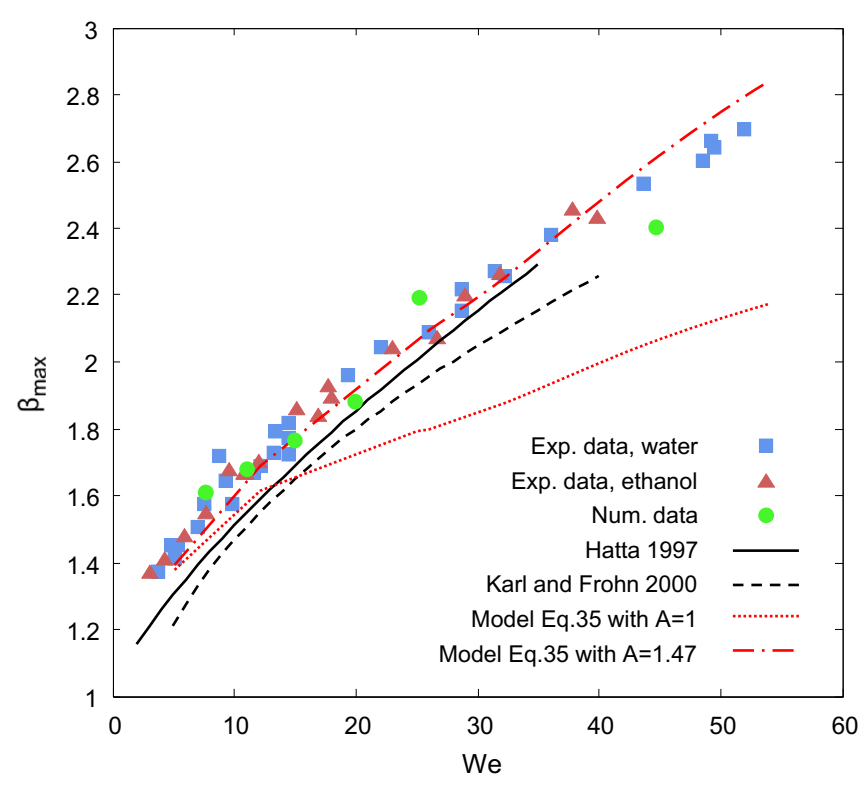

Fig. 10. Maximum Spreading Diameter versus the normal Weber number. Comparison between the present numerical simulations, the correlations in [31,38], the model of Eq. (35) and the present experiments.

neighborhood large enough to cover the region where the grid is very refined when the droplet is near the hot surface $[3,23,65]$. Finally, the simulation requires a specific model of phase change which deals with both evaporation and boiling since the two occur simultaneously (see Section 5).

For the $\mathrm{We}=7.4$, the simulations are performed with two computational grids $96 \times 192$, and $144 \times 288$. The grids are nonuniformed and strongly refined in the neighborhood of the solid surface. The radial direction is decomposed in two parts: a uniform zone between the radial coordinates $x=0$ and $x=L_{r} / 2$ with 60 for the coarsest grid and 90 points for the thinnest grid, and a continuously stretched zone until $x=L_{r}$ which contains respectively 36 and 54 grid cells. In the longitudinal direction, the grid can be also decomposed in two regions: the very refined zone between the coordinates $z=-L_{z} / 2$ and $z=-L_{z} / 2+\epsilon$ with $\epsilon=0.06 L_{z}$ containing respectively 40 and 80 grid cells, and a uniform region in the rest of the domain which contains 152 and 208 points for the coarsest and the thinnest grids respectively. The transition between all the different parts is continuous in order to preserve the accuracy of the spatial discretization.

Table 5 reports quantitative comparisons between the present numerical simulations and the corresponding experimental measurements obtained from high-speed shadowgraphy (as mentioned in Section 3). The table shows that the numerical simulations and the experimental data are in good agreement, especially for the the radial diameter at the maximum spreading (or spreading diameter) $\beta_{\max }$ and the velocity ratio $r_{n}$ since the differences are respectively around $12 \%$ and $7 \%$. The simulations are well resolved since there is little difference between the results performed with the two grids. Fig. 6 shows indeed almost superimposed evolutions of the spreading diameter for the simulations performed with the two grids.

\subsection{Droplet shape and satellite drops}

We first compare the evolution of the droplet shape between the experiments and the numerical simulation. Fig. 7 shows that the deformation of the droplet is well reproduced in the simulations. As expected, the spreading diameter is higher for increasing
Weber numbers since the inertial effects increase in comparison to the capillary ones. Furthermore, the numerical simulations accurately predicts the formation of a satellite droplet for the $W e=44.8$ impact. This phenomenon was thoroughly studied by Karl and Frohn in [38] for water and ethanol droplets with diameters ranging from 70 to $260 \mu \mathrm{m}$. This study covers different impact regimes from reflection (bouncing without satellite drop) to splashing (desintegration into numerous small droplets) through satellite droplet formation (one to three satellite drops) and is focused on the transition between the bouncing regime and the satellite droplet formation. The authors predict the transition for normal Weber numbers ranging from 20 to 30 and for an empirical parameter $C_{K F}=W e R e^{0.8}$ higher than 2000 . In the present investigations, the experiments allow to determine the onset of the regime of satellite droplet formation. Images of four monodispersed droplet streams impacting the surface at $W e=25.2$ are presented in Fig. 8. Frame 3 of Fig. 8 show the formation of a satellite droplet while on frames 1,2 and 4 , the surface tension is able to maintain a closed surface. Therefore, the transition takes place for We $\sim 25$ and $C_{K F} \sim 3255$ which is consistent with the experimental results from [38]. Nevertheless, it should be noticed that the droplet does not break up after the bouncing in the numerical simulation of the impact at $\mathrm{We}=25.2$, even if it is very close to.

\subsection{Spreading diameter}

To determine the parameters that predominantly control the deformation, Fujimoto et al. investigated the droplet/wall interaction for different diameters and wall temperatures [20]. For surface temperatures below the Leidenfrost point, the Reynolds and the Weber numbers control the phenomenon while for surface temperatures above $T_{\text {Leid }}$, the deformation of the droplet depends mostly on the Weber number. In Fig. 9a the dimensionless spreading factor $\beta$ against the dimensionless time $t^{*}$ is plotted for different Weber numbers, using the following definitions for the parameters $\beta$ and $t^{*}$ :

$\beta=\frac{D_{r}}{D_{0}} \quad$ and $\quad t^{*}=t \frac{U_{0}}{D_{0}}$

where $D_{r}$ is the maximum diameter of the droplet along the transversal direction to the wall and $U_{0}$ is the droplet initial velocity normal to the wall. Fig. 9 highlights the different stages of the droplet deformation. Basically, the deformation process can be divided into three consecutive phases: kinematic, spreading, and recoiling. During the kinematic phase, the bottom of the drop is stopped at impact, but the upper part of the drop is still moving with an undisturbed velocity so that the drop looks like a cut sphere. Typically, the kinematic phase is limited to $t^{*}<0.1$. Since the spreading lamella is not yet visible in the kinematic phase, $\beta$ remains equal to 1 . In the spreading phase, a lamella is ejected from the base of the drop and forms a thin film with a bounding rim because of the capillary effects. After the spreading phase, the drop begins to recede radially. The recoil is initiated by the dominating surface forces which restore the drop shape minimizing the surface energy. Finally, the elongated droplet is bouncing and its surface oscillates. As illustrated in Fig. 9a, the drop evolution in the spreading and recoiling phases is clearly a function of the normal Weber number. Numerical and experimental results are in good agreement for the spreading phase. In particular, both give very close values of $\beta_{\max }$. However, the receding is significantly slower in the experiments. A possible explanation for this discrepancy in the recoiling phase could be made by considering the influence of the tangential velocity of the droplets in the experiments. As opposed to the simulations that do not consider any tangential motion due to the axisymmetric assumption, in the experiments, the tangential 


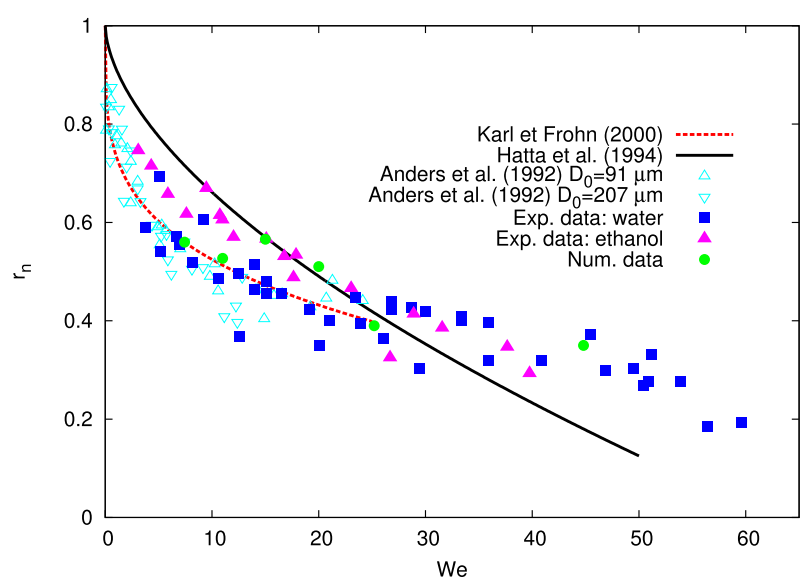

Fig. 11. Velocity ratio versus the normal Weber number. Comparison between the present numerical simulations, the correlation in [38], the present measurements and experimental results from [2].

velocity could induce an additional shear stress along the tangential direction to the wall, slowing down the interface during the recoiling phase. Another possibility relates to the change in temperature which becomes significant in the recoiling phase. The heating of the liquid results in a variation of the surface tension and the liquid viscosity. This effect is not taken into account in the simulations, which assume constant properties.

In the past, approaches based on the balance of mechanical energies have been frequently used to model the spreading of droplets. In these approaches, no liquid motion is considered at the time of maximum spread, meaning that all the initial mechanical energy is either dissipated or transformed into surface energy. Due to the lack of knowledge concerning the flow field inside the drop, the viscous dissipation is usually incorporated in the models by making some basic assumptions. By performing an energy balance analysis considering the viscous dissipation to be half the initial kinetic energy and the droplet to have a cylinder-like shape at the maximum spreading, Karl and Frohn deduced the following formulae:

$\beta_{\max }^{3}-\left[\frac{W e}{6}\left(\frac{1+r_{n}^{2}}{2}\right)+2\right] \beta_{\max }+\frac{4}{3}=0 \quad 0 \leqslant W e \leqslant 55$

$r_{n}=1-0.263 W e^{0.257} \quad 0 \leqslant W e \leqslant 25$

where $r_{n}$ is the ratio of the normal velocity before the impact to the normal velocity after the impact. Emperical correlations have been also formulated to provide information on how $\beta_{\max }$ is increased by the Weber number for a given liquid which is generally water. The correlation of Hatta et al. based on the experimental results from [32] with water droplets impinging onto an inconel alloy surface, can be expressed as follows:

$\beta_{\max }=0.093 W e^{0.74}+1 \quad 0 \leqslant W e \leqslant 45$

It is shown in [31] that experimental results of Ueda et al. and Akao et al. $[1,66]$ are in good agreement with Eq. (32). Fig. 9a shows that the maximum spreading diameter increases with the Weber number. The same tendancy is observed in Fig. 10 which reports the results of the present numerical simulations, the present experimental data, and the correlations of Karl and Frohn and Hatta et al. $[16,14,31,38]$. Fig. 10 shows a good agreement between numerical simulations, experiments and correlations. The correlation of Hatta et al. seems to be a better approximation for the present experimental and numerical results. The numerical results are also reassuringly in line with the experimental measurements. It should be emphasized that Karl and Frohn consider the diameter

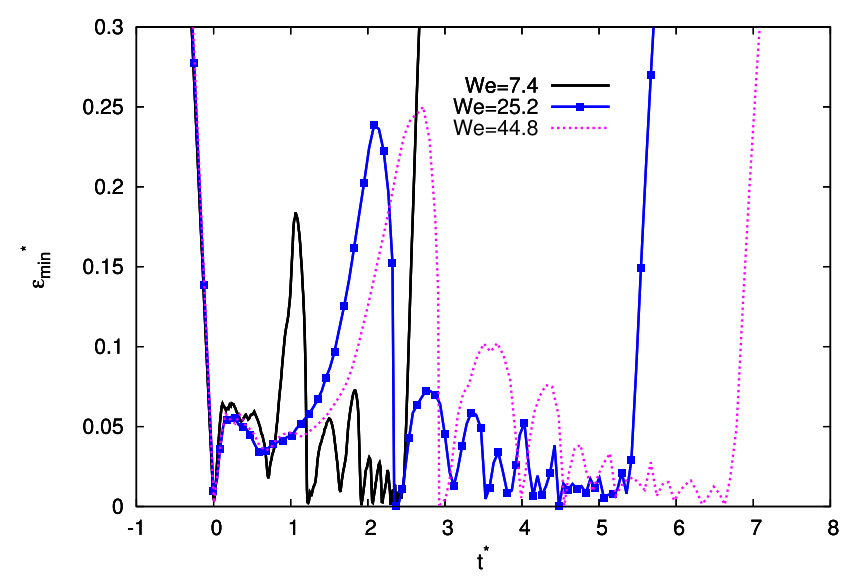

Fig. 12. Minimum vapor layer thickness $\varepsilon_{\min }^{*}=\varepsilon_{\min } / D_{0}$ during the droplet/wall interaction versus the dimensionless time $t^{*}$ for different Weber numbers (numerical results).

of the apparent contact area below the droplet to derive the model (30). The diameter of the contact area is smaller than the spreading diameter, the difference corresponding to the rim surrounding the spreading lamella.

As an alternative to the approaches mentioned above, Roisman et al. in [52,53] suggested a method based on the resolution of the mass and momentum equations applied to the rim bounding the spreading droplet. The method initially devised to describe the drop impact in isothermal conditions [53] and the binary collision of droplets [52], was recently implemented by Castanet et al. [10] in the film boiling regime. Only the main equations are recalled in the following paragraph. Provided sufficiently large Reynolds and Weber numbers, the spreading droplet rapidly forms a thin and smooth liquid sheet. At the large times, the velocity of the liquid in this sheet can be approximated by $[10,52]$ :

$u_{r}=\frac{A r}{t^{*}+\tau}$

where all the parameters are made dimensionless by $D_{0}$ and $U_{0}$. In this expression, $A$ and $\tau$ are two integration constants. The inviscid remote solution formally derived by Yarin and Weiss [70] corresponds to $A=1$. Using numerical simulations, Roisman et al. [51] verified that $A$ is actually close to 1 when a droplet impinges onto a wetting surface and the frictions exerted on the liquid at the solid surface are neglected. Based on these simulations, they could also propose the following expression for the lamella thickness:

$h=\frac{\eta}{\left(t^{*}+\tau\right)^{2}} \exp \left(-\frac{6 \eta r^{2}}{\left(t^{*}+\tau\right)^{2}}\right)$

where $\eta=0.39, \tau=0.25$. According to Castanet et al. [10], the conservation of momentum applied to the rim yields:

$W_{R} \ddot{R}_{R}=2 \pi R_{R} h_{L}\left(\left[u_{\text {rim }}-\dot{R}_{R}\right]^{2}-\frac{6}{\operatorname{Re}\left(t^{*}+\tau\right)}-\frac{2}{W e h_{L}}\right)$

where $R_{R}$ is the position of the center of mass of the rim divided by $D_{0}, h_{L}=h\left(R_{R}\right)$ and $u_{\text {rim }}=A R_{R} /\left(t^{*}+\tau\right)$. The volume of the rim $W_{R}$ can be deduced from the mass conservation using Eq. (34). Eq. (35) was solved numerically using the same approach as Castanet et al. [10].

Table 6

Time-average minimum vapor layer thickness.

\begin{tabular}{cccc}
\hline We & 7 & 25 & 45 \\
\hline $\bar{\varepsilon}_{\min }(\mu \mathrm{m})$ & 3.86 & 4.40 & 4.75 \\
\hline
\end{tabular}


The spreading evolutions calculated with $A=1$ are presented in Fig. 9b-d. It appears that the inertial force acting onto the rim (the term in $\left(u_{\text {rim }}-\dot{R}_{R}\right)^{2}$ in Eq. (35)) is not sufficient to match the experimental and simulated data with the model. This means that the value of $A$ is certainly enhanced in the film boiling regime compared to the impact on a dry wetting surface. This conclusion was also drawn by Castanet et al. [10], who suggested a value of $A$ equal to 1.47 in the case of water. As displayed in Figs. 9 and 10, this higher value of $A$ allows obtaining a good agreement between the model and the experimental and numerical data. To justifiy the increase of $A$, Castanet et al. [10] evaluated the shear stress exerted by the vapor flow on the liquid interface beneath the droplet. They found out that the vapor was able to entrain the liquid in the lamella due to a large radial velocity. They also pointed out that the velocity field inside the lamella may take a longer time to converge to the asymptotic remote solution given in Eq. (33) in the presence of this accelerating force acting on the liquid which does not exist in the impact onto a wetting surface.

\subsection{Loss of momentum}

The loss of momentum is caused by the internal dissipation during the droplet deformation. Experimental studies of droplet impinging onto hot surfaces with different impinging angles $[2,14,38]$ show that the loss of momentum in the tangential direction to the surface $L_{t}$ can be neglected $(\sim 5 \%)$. Therefore, only the loss of momentum in the normal direction to the surface, $L$, is considered presently. In [38], the authors suggested that the internal velocity within the droplet mainly governs the phenomenon. This velocity depends on the fluid inertia which is characterized by the Weber number. In $[2,38]$, the authors quantify the loss of momentum $L$ with the velocity ratio $r_{n}$ define as follows:

$L=\frac{m_{b} \bar{V}_{b}-m_{a} \bar{V}_{a}}{m_{b} \bar{V}_{b}}=1-\frac{m_{a} \bar{V}_{a}}{m_{b} \bar{V}_{b}}$

$L \simeq 1-r_{n}$

where the subscripts $b$ and $a$ stand for before and after the impact, $\bar{V}$ is the average droplet velocity and $m$ the droplet mass. The mass loss due to the vaporization of the droplet during the impact can be neglected $\left(m_{b} \simeq m_{a}\right)$ so Eq. (36) leads to Eq. (37). Hatta et al. show that according to their experimental data, the evolution of the velocity ratio is described by the following correlation:

$r_{n}=1-0.087 W e^{0.59}$

The evolution of the non-dimensional loss of momentum with the normal Weber number is reported in Fig. 11 for the correlation of Karl and Frohn ( $c f$ Eq. (31)), the experiments by Anders et al. [2], the present experimental data [14], and the present numerical data. These results are in excellent agreement whereas the correlation of Hatta et al. (Eq. (38)) differ from the others [32]. One explanation of this difference may be the way the velocity is computed, since Hatta et al. defined $\bar{V}_{a}$ to be the average value between the velocity at the top of the droplet and the velocity at the bottom whereas for the rest of the studies $\bar{V}_{a}$ is the average velocity of the droplet. The figure shows that the velocity ratio decreases when the normal Weber number increases. The loss of momentum can reach $60 \%$ for Weber numbers higher than 35 . It is worth

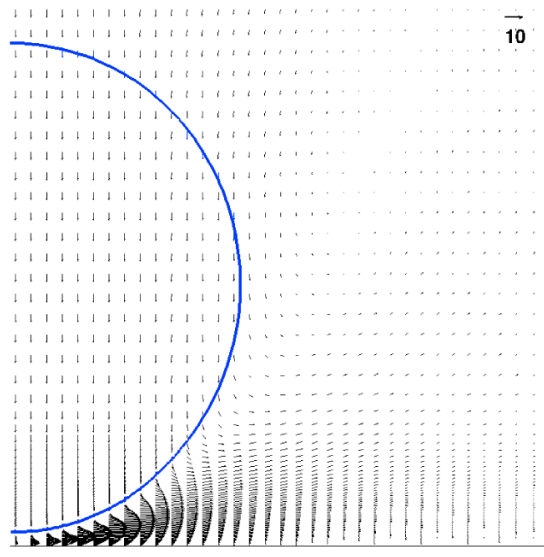

1.

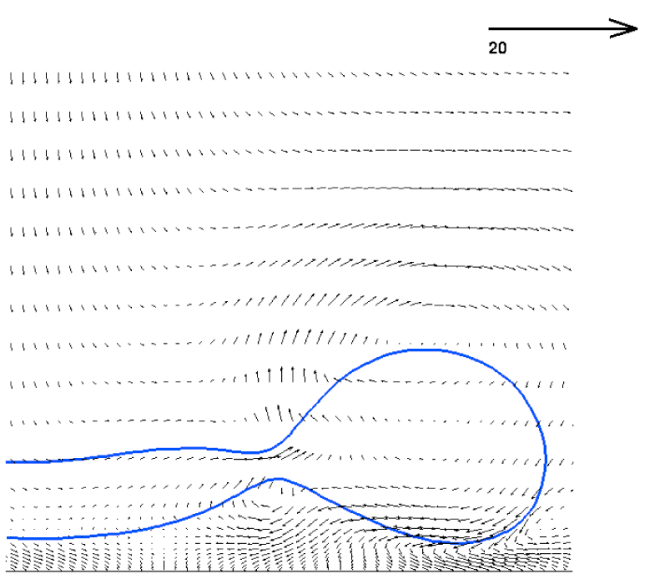

2.

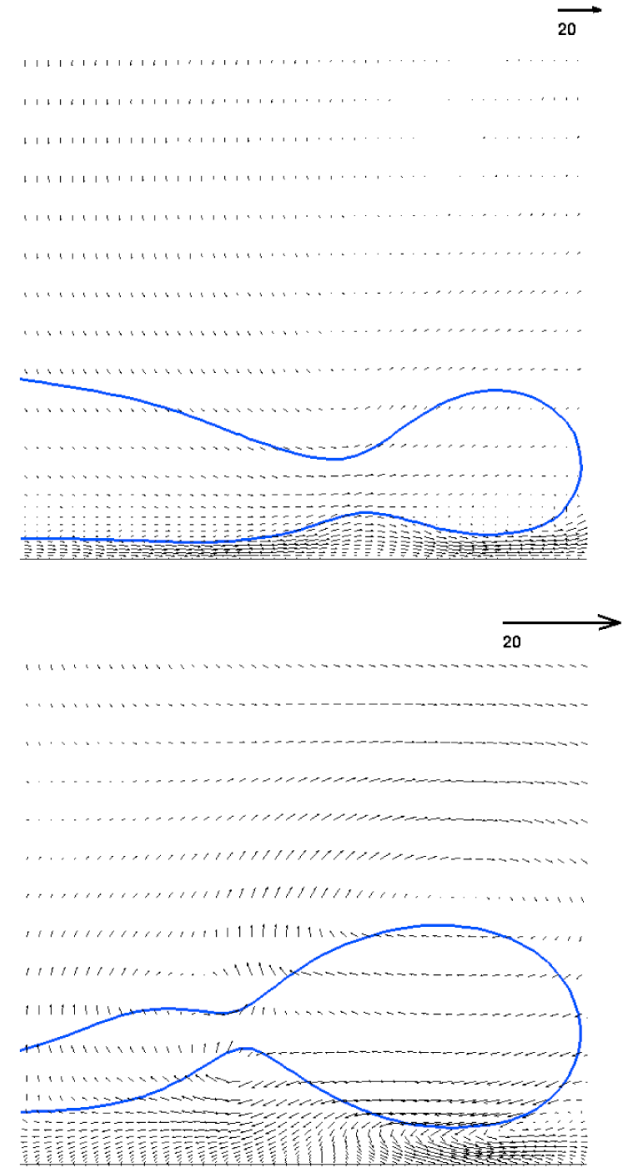

Fig. 13. Velocity field and interface evolution during the droplet interaction with the hot surface with $W e=25.2$. 


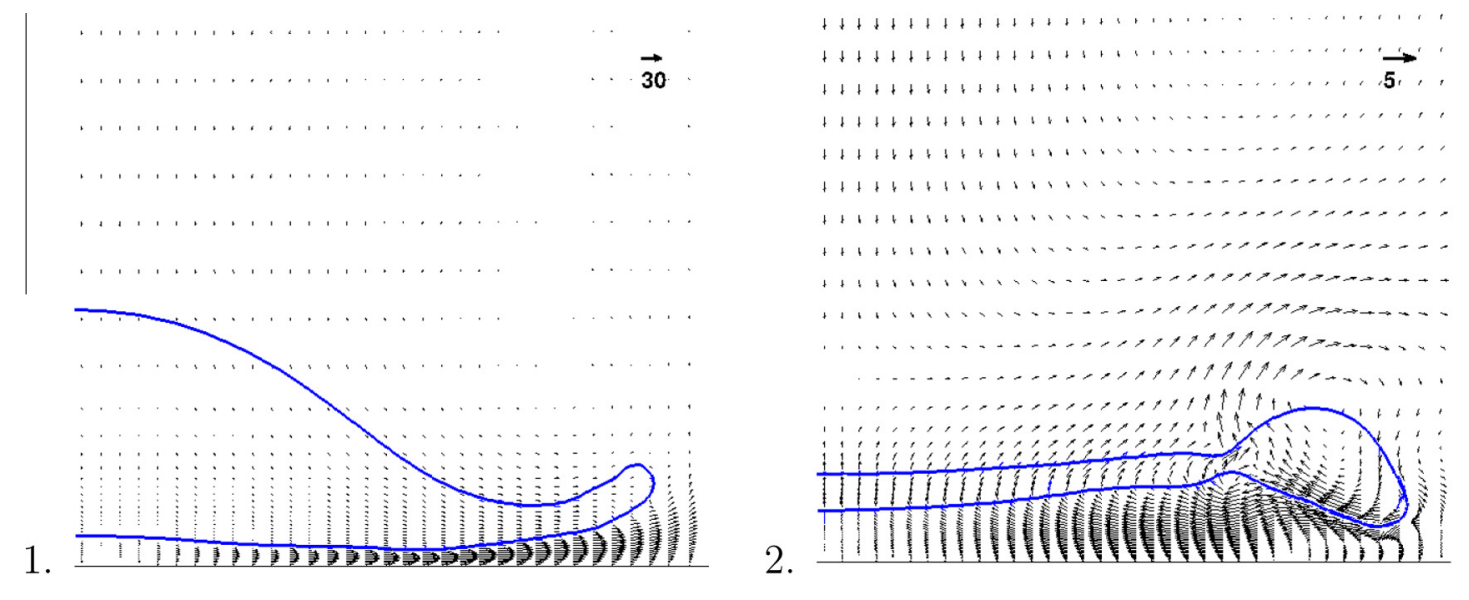

Fig. 14. Velocity field and interface evolution during the droplet interaction with the hot surface with $W e=44.8$.

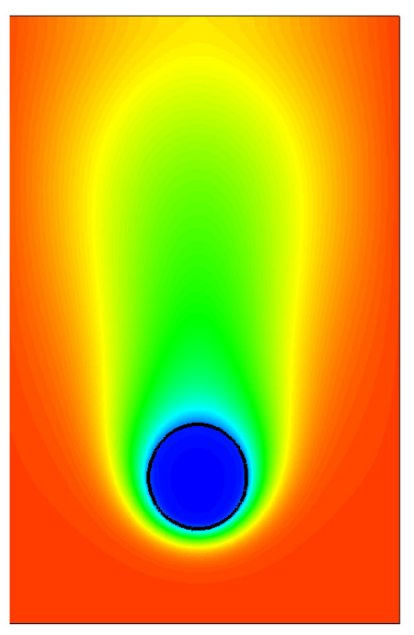

1.

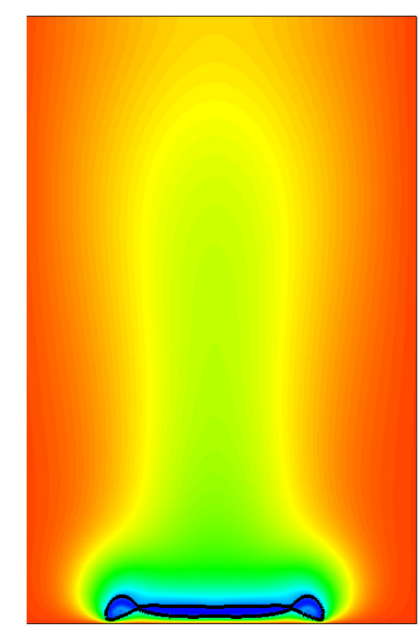

3.

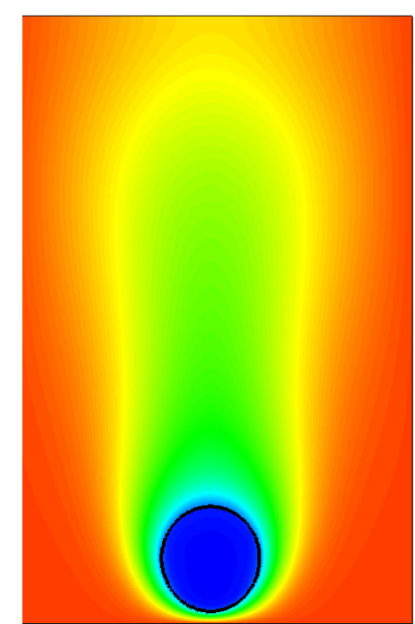

2 .

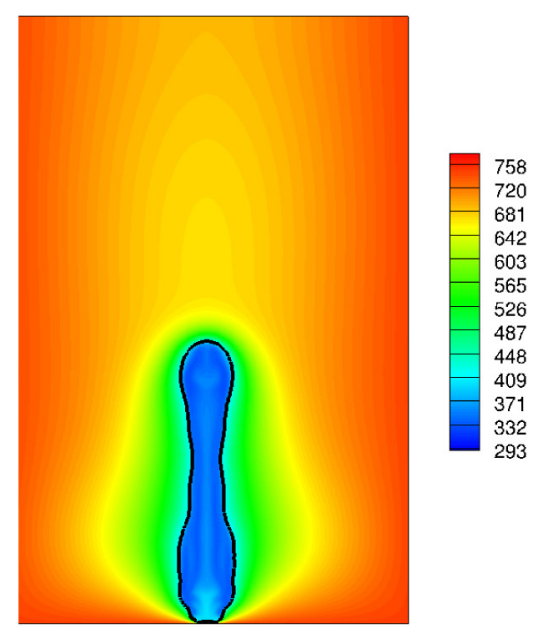

4.

Fig. 15. Temperature field and interface evolution during the droplet interaction with the hot surface with $W e=25.2$.

noting that the experiments were performed with different impinging angles $\alpha\left(0^{\circ} \leqslant \alpha \leqslant 50^{\circ}\right.$ and $\left.\alpha=90^{\circ}\right)$ and different surface temperature $\left(240{ }^{\circ} \mathrm{C} \leqslant T_{w} \leqslant 873^{\circ} \mathrm{C}\right)$. These parameters do not seem to have a major influence on the loss of momentum.

\subsection{Vapor layer dynamics}

The thickness of the vapor layer, which insulates the droplet from the solid surface, is varying with the droplet diameter and 


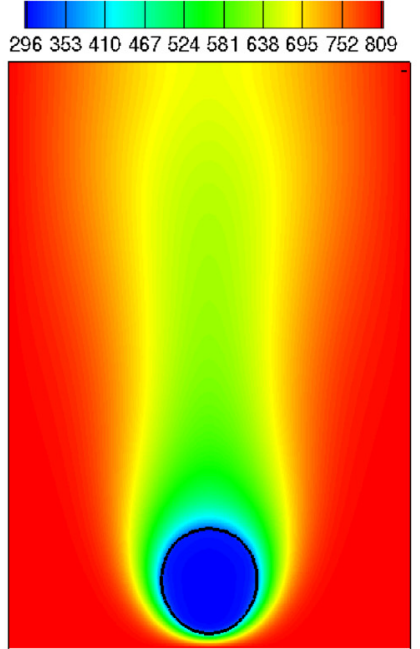

1.

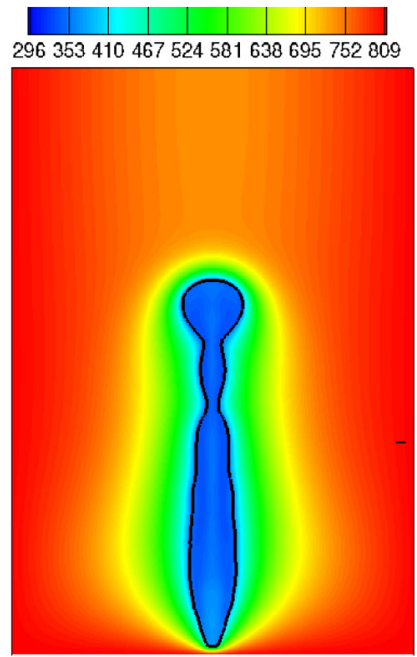

3.

Fig. 16. Temperature field and interface evolution during the droplet interaction with the hot surface with $W e=44.8$.

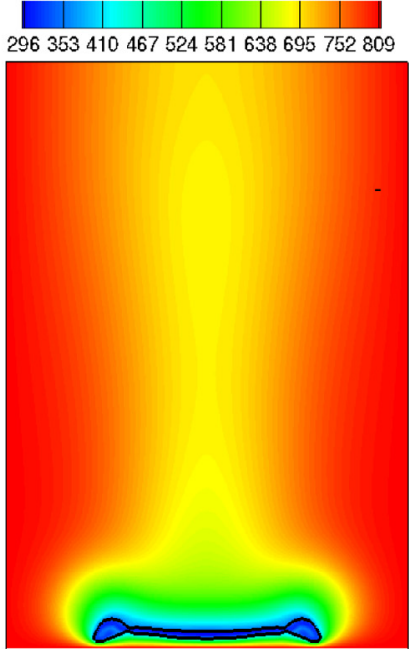

2.
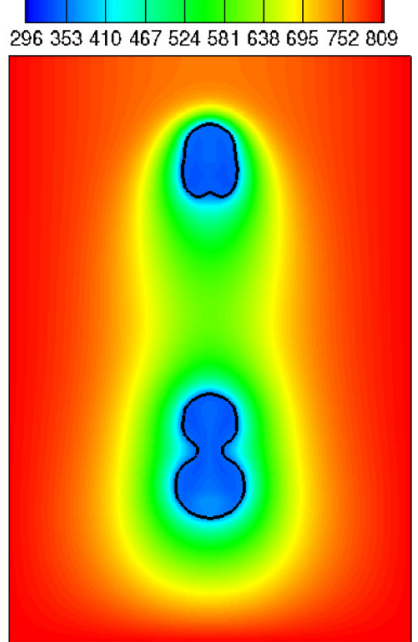

4. the heat flux. It is several orders of magnitude smaller than the droplet initial diameter $[6,8,21]$. Experimentally, it is quite difficult to fully access to relevant information on the vapor layer. To our knowledge, all the experiments where the vapor layer thickness $\varepsilon$ was measured, were performed with sessile droplets sizing a few millimeters. In this situation, the film thickness is large enough for a rear illumination to pass under the drop [6,8]. In [8], the authors show that the vapor layer beneath the droplet forms a concave pocket and consequently $\varepsilon$ depends on the position of the interface. In their study, the minimum thickness, $\varepsilon_{\min }$, ranges from $5 \mu \mathrm{m}$ to $100 \mu \mathrm{m}$ when the drop size varies from $0.5 \mathrm{~mm}$ to $7 \mathrm{~mm}$. Also these estimates are obtained for a surface at $643 \mathrm{~K}$ and droplets at the saturation temperature. Similarly, Biance et al. show that for a hot surface at $573 \mathrm{~K}$ and $D_{0}$ between $1.7 \mathrm{~mm}$ and $7 \mathrm{~mm}, \varepsilon_{\min }$ ranges from $30 \mu \mathrm{m}$ to $100 \mu \mathrm{m}$. For this range of initial diameter, the vapor layer thickness seems to increase with $D_{0}$. It is worth noting that the value of $\varepsilon_{\min }$ for small droplets $\left(D_{0}=0.5 \mathrm{~mm}\right)$ and larger droplets $\left(D_{0}=7 \mathrm{~mm}\right)$ differ of almost two orders of magnitude. Furthermore, using a lubrication approximation and assuming that there is a radial Poiseuille flow in the vapor layer, Biance et al. deduced two formulae to calculate $\varepsilon_{\min }$, provided that the droplet diameter is larger or smaller than the capillary lenght $\lambda_{c}$ (Eqs. (6) and (8) in [6]). Based on these formula, the vapor layer thickness is found to be about $3.8 \mu \mathrm{m}$ at $\mathrm{We}=7.2$. This value is comparable to the present numerical results reported in Fig. 12.

There are very few numerical studies of the impact of droplets on heated surface with temperatures above the Leidenfrost point as it is very challenging to capture the dynamic of the flow inside a thin vapor film. In $[21,29,30]$, the authors used a vapor layer model to determine the dynamics, the evaporation rate and the heat transfer in the vapor layer, and the Navier-Stokes equation to compute the moving droplet and its deformation. The evaporation and the heat transfer outside of the vapor layer are then neglected. For instance, Ge and Fan performed simulations of the impact of subcooled droplets onto a hot surface and determined the vapor layer thickness for different degree of subcooling $\left(T_{\text {sat }}-T_{\text {drop }}=2{ }^{\circ} \mathrm{C}, 30^{\circ} \mathrm{C}\right.$ and $\left.88^{\circ} \mathrm{C}\right)$. They show that the vapor layer thickness decrease with the degree of subcooling since it is approximatively inverse proportional to the heat flux at the solid surface. For a small subcooling degree $\left(2{ }^{\circ} \mathrm{C}\right)$, the thickness of the vapor layer varies from 3 to $35 \mu \mathrm{m}$ during the impact period whereas for a higher subcooling degree $\left(88^{\circ} \mathrm{C}\right)$ it remains very small and almost constant $(\sim 0.5 \mu \mathrm{m}-1 \mu \mathrm{m})$. According to them, for a high subcooled degree, most of the thermal energy is spent to heat the droplet whereas for a small subcooled degree, almost 

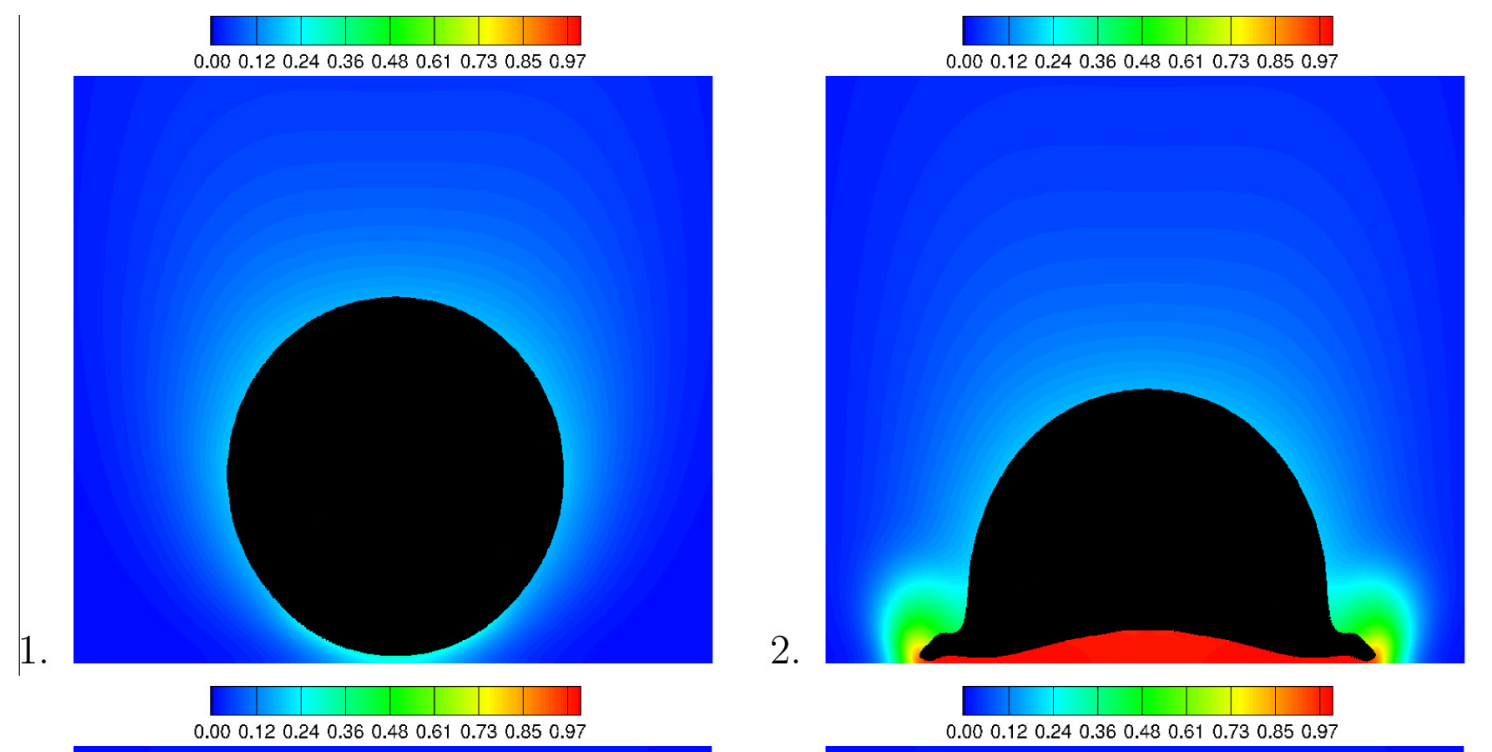

3.

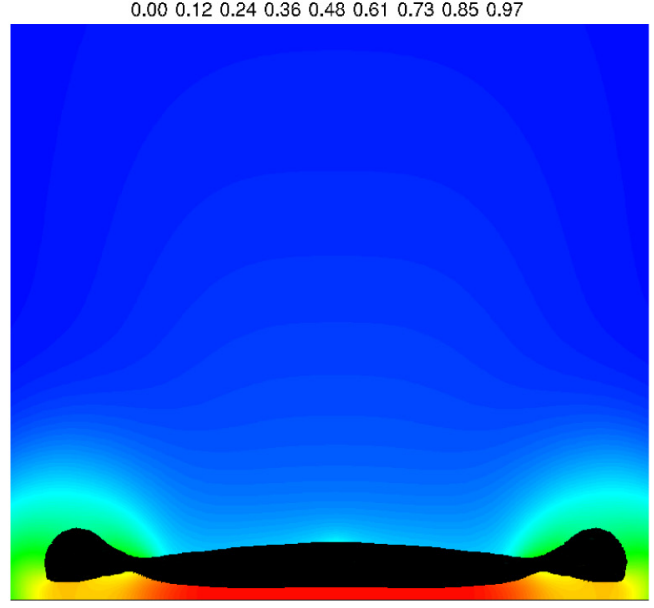

4.

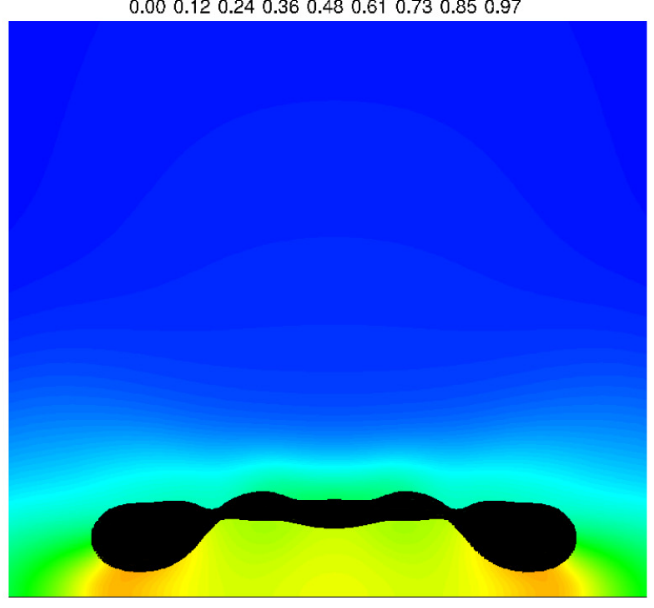

Fig. 17. Mass fraction field and interface evolution during the droplet interaction with the hot surface $(W e=25.2)$.

all the thermal energy is used to evaporate the droplet leading to a higher evaporation rate. In the present investigation, the temporal evolution of the minimum vapor layer thickness has been evaluated for a subcooling degree of $78^{\circ} \mathrm{C}$. The results reported in Fig. 12 show that $\varepsilon_{\min }$ oscillates over time from less than $0.1 \mu \mathrm{m}$ to $14 \mu \mathrm{m}$. The time-average vapor layer thickness, $\bar{\varepsilon}_{m i n}$, is reported in Table 6 for $W e=7, W e=25$ and $W e=45$. It slightly increases with the Weber number. It is worth noting that in the numerical simulations, the droplet never enters into contacts with the hot surface. In Fig. 12, we can observe an oscillatory behavior of the minimum vapor layer thickness. The frequency of this oscillation increases with time. The first major peak corresponds to the beginning of the receding process. That can be explained by considering that most of the impacting kinetic energy of the droplet has been transformed into surface energy. Therefore, the film thickness results mainly from a force balance between the droplet weight and the pressure force in the vapor layer. Next during the receding phase several minor peaks are observed with an increasing frequency and decreasing amplitude. These oscillations are due to the capillary waves during the receding process. As the spreading diameter decreases with time, the wavelength of this capillary waves also decreases and it leads to smaller amplitudes and higher frequencies.

Many studies state that the flow in the vapor layer is approximatively a radial Poiseuille flow $[4,6,9,20,24,38,71]$. As very refined grids are used in this specific zone of our computation domain, the simulations are able to resolve the smallest scales of this flow. Indeed, a strongly refined grid near the solid surface is used in order to ensure that several grid cells allow to compute the thermal and dynamical effects along the $z$ direction in the vapor layer. The velocity field during the droplet/surface interaction is reported in Figs. 13 and 14 corresponding respectively to the cases $W e=25.2$ and $W e=44.8$. In order to have suitable visualization of the velocity field the vectors are plotted every 3 points along $\overrightarrow{e_{z}}$. In Fig. 13, the first picture is taken before the impact, the second during the droplet spreading and the two last ones during its receding. In the first frame of Fig. 13, the velocity in the vapor layer is high $\left(>10 m \cdot s^{-1}\right)$. This is in line with thermal gradients in the vapor layer leading to a high evaporation rate. In the second frame of Fig. 13 and the first frame of Fig. 14, the droplet almost reaches its maximum spreading and an almost radial Poiseuille Flow can be observed in the vapor film. After reaching the maximum spreading, the droplet begins to recoil from the surface. In frame 3 of Fig. 13, it can be observed that the droplet receding is accompanied by a flow recirculation in the deflated zone of the droplet (at the junction between the lamella and the rim) due to the motion of the liquid interface. In the vapor layer under the rim, the velocity near the interface and the velocity close to the solid surface have opposite directions. Finally, in the fourth frame of Fig. 13 and frame 2 of the Fig. 14, the flow recirculation remains under the rim, while 


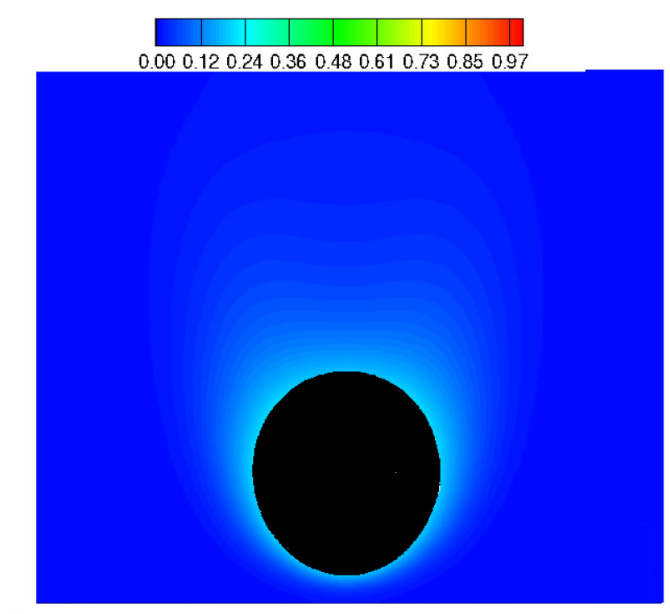

1.

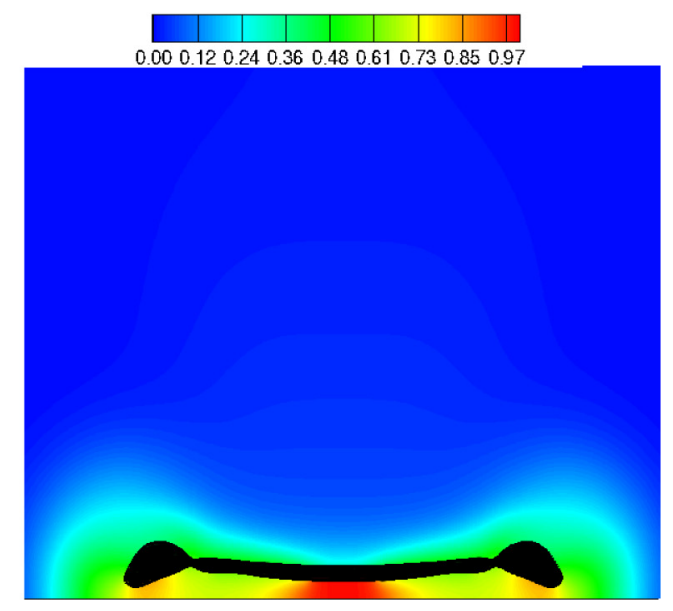

3.

Fig. 18. Mass fraction field and interface evolution during the droplet interaction with the hot surface $(W e=44.8)$.

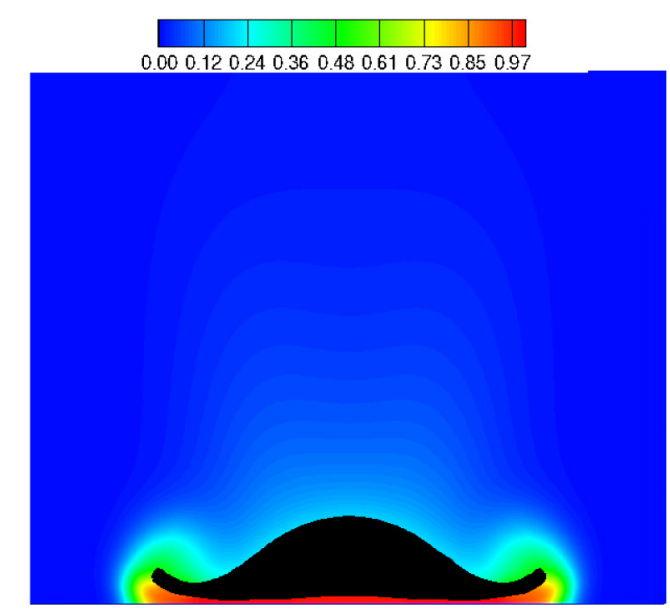

2.

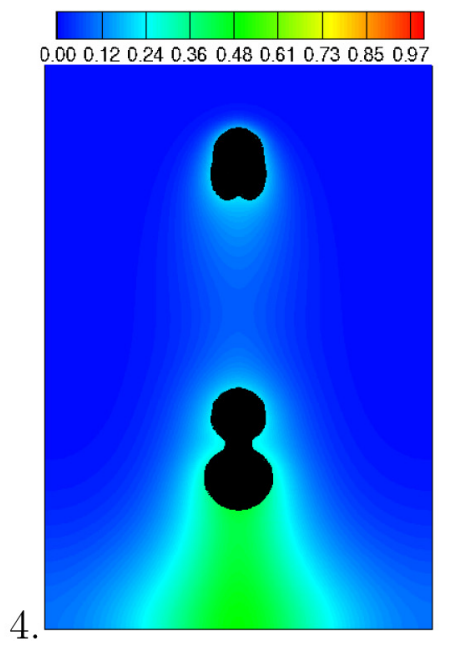

there is a radial inward flow under the rest of the droplet. The velocities obversed in the vapor film during the receding are about twice as small as in the spreading phase.

\section{Heat and mass transfer}

When a droplet impacts a heated surface above the Leidenfrost point, the temperature at the interface between the droplet and the surrounded gas can locally reach the boiling point. Evaporation also occurs spontaneously wherever the vapor is not saturated. This section is devoted to the study of the transient vaporization regime and the resulting thermal transfer between the liquid phase and the gas phase.

\subsection{Temperature field in the gas phase}

In the numerical simulations, a water droplet initially at the ambient temperature $\left(T_{\text {drop }} \sim 290 \mathrm{~K}\right)$ is moving in a hot gas $\left(T_{g} \sim 800 \mathrm{~K}\right)$. The variation of the surface temperature during the impact mainly depends on the residence time, the droplet temperature and the thermal effusivity of the surface material. For instance, for an inconel surface, variations can reach $25 \mathrm{~K}$ for a subcooling degree of $30 \mathrm{~K}[21,35]$. However, in the present study, the decrease of the wall temperature is neglected, which is tantamount to the use of a surface material with a high thermal effusivity. As shown in Fig. 15 (frames 3 and 4) and Fig. 16 (frames 2 and 3), the thermal gradient into the vapor layer is much higher that the thermal gradient in the liquid. Indeed, the temperature in the vapor film of a few microns thickness is varying from $\sim 373 \mathrm{~K}$ at the interface between the droplet and the air, to $800 \mathrm{~K}$ at the solid surface.

\subsection{Evolution of the mass fraction field of vapor during the impact}

The mass fraction field in the gas phase is reported in Figs. 17 and 18 for $W e=25.2$ and $W e=44.8$. In the liquid phase, shown in black in Figs. 17 and 18, $Y_{\text {vap }}$ is equal to 1 since the liquid is assumed to be pure. In the first pictures (frame 1 of Figs. 17 and 18 ) before the spreading, the droplet evaporates slowly and the vapor diffuses into the gas phase. It can be observed in Fig. 17 (frame 2) and Fig. 18 (frame 2) that during the spreading of the droplet, the vapor layer is saturated in liquid vapor. The droplet evolves from a purely evaporating regime to a mixed regime in which temperature of the liquid at the interface with the vapor film reaches the boiling point whereas the rest of the droplet surface is still evaporating (frames 3 and 4 of Figs. 19 and 20).

After reaching $\beta_{\max }$, during the receding of the droplet, the vapor layer thickness increases. Frames 3 and 4 of Fig. 17 and frame 3 of Fig. 18 show that the vapor layer is saturated during the droplet spreading whereas during the receding phase, it is not the case anymore. Indeed, a significant amount of outside gas is entrapped in the thin vapor layer due to the receding motion and is mixed with the vapor. In Fig. 13 (frames 3 and 4) and Fig. 14 (frame 2) 

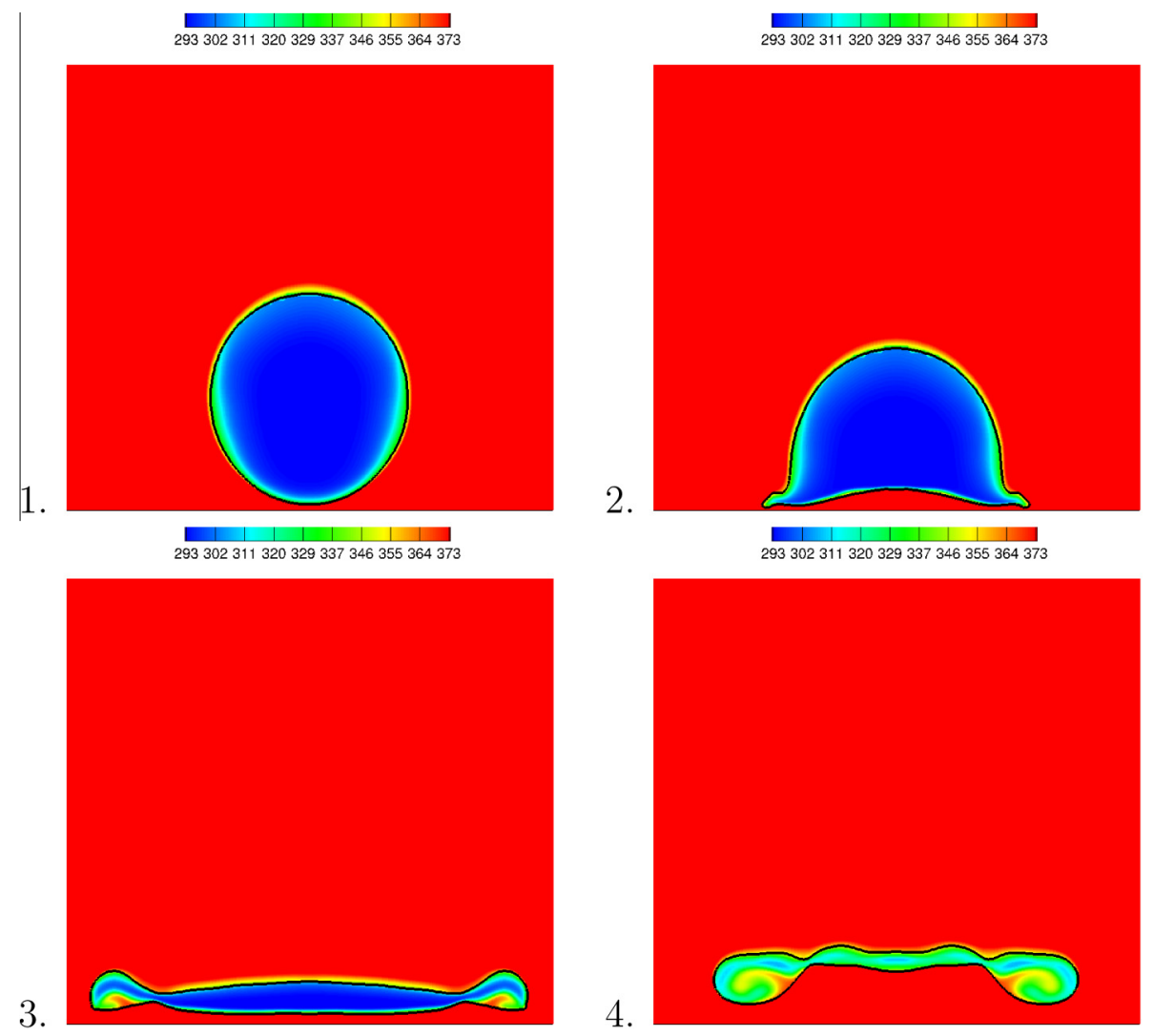

Fig. 19. Temperature field in the liquid phase and interface evolution during the droplet interaction with the hot surface $($ We $=25.2)$.

it appears that an inward velocity field below the droplet rim brings air into the vapor layer. The latter is no longer saturated in vapor and a transient regime of vaporization is captured instead by the present numerical method. Finally, an image of the droplet leaving the surface is reported in Fig. 18 (frame 4).

\subsection{Evolution of the temperature field inside the droplet during the impact}

Some snapshots of the liquid temperature field during the drop impact are reported in Figs. 19 and 20 for $\mathrm{We}=25.2$ and $\mathrm{We}=44.8$. At the first times of the spreading (frame 2 of Figs. 19 and 20), the isocontours of the temperature are roughly parallel to the droplet surface, indicating that heat conduction is dominating the transfers. In contrast, the temperature pattern, especially in the rim, is somewhat more complex during the receding (frames 3 and 4 of Figs. 19 and 20). This suggests that the internal liquid motions eventually play an important role as they significantly accelerate the mixing.

The increase in droplet temperature during the impact was estimated at different surface temperatures and Weber numbers. The results partially published in [16] are compared to the present numerical data in Fig. 21. The experimental data show that the droplet heating is nearly independent of the wall temperature in the Leidenfrost regime. In contrast, the droplet heating increases with the Weber number. This experimental observation is corroborated by the present numerical results, but the droplet heating in the simulations is larger in the case of $\mathrm{We}=44.8$. The uncertainty of the experimental data alone (typically of a few degree as suggested by the scattering of the data in the figure) cannot explain this difference which may result from the accumulation of several factors. First, in the numerical simulations, the surrounded gas is initially considered to be at the same temperature as the solid surface prior to the droplet passage. This hypothesis is inaccurate: as a matter of fact, away from the hot surface, a thermal gradient related to the natural convection is established. Therefore, the imposed initial and boundary conditions may contribute to overestimate the heating of the droplet in the numerical simulations. However, their influence is limited since the heating of the droplet occurs mostly during the interaction with the surface (frames 3 and 4 of Fig. 19 and 2 and 3 of Fig. 20).

Another difference between the numerical simulations and the experiments lies in the tangential velocity of the droplets in the experiments. The experiments were performed with different incident angles whereas the numerical simulations are axisymmetric. In $[38,68]$, the authors show that for small impact angles, the air can be driven into the vapor layer. The tangent velocity due to the impact angle is likely to deplete the vapor in the film. This effect contributes to further increase the thickness of the film layer, to cold down the gas in it, and thus to reduce the droplet heating. It should be also emphasized that, in the present computational model, the flow is considered to be incompressible and the parameters, such as viscosity and density, to be constant. Given the difference of temperature between the liquid and the surface which are just a few micrometers apart, a significant change of the fluids properties is likely and the consequences on the droplet heating can be questioned.

\subsection{Thermal transfer}

In the following, we introduce the vaporization energy $E_{v}$, which is the heat taken by the liquid to evaporate, and $E_{\Delta T}$ the heat entering the droplet. These dimensionless parameters are defined as follows: 

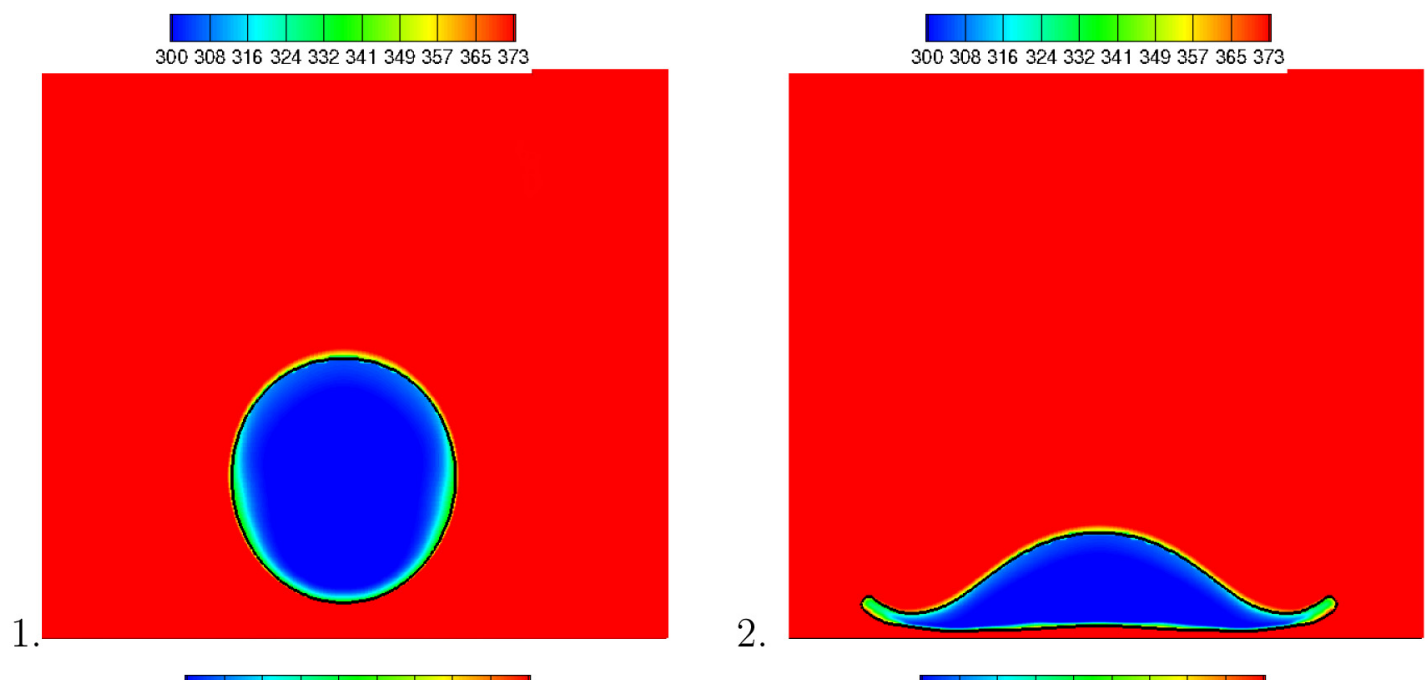

3.
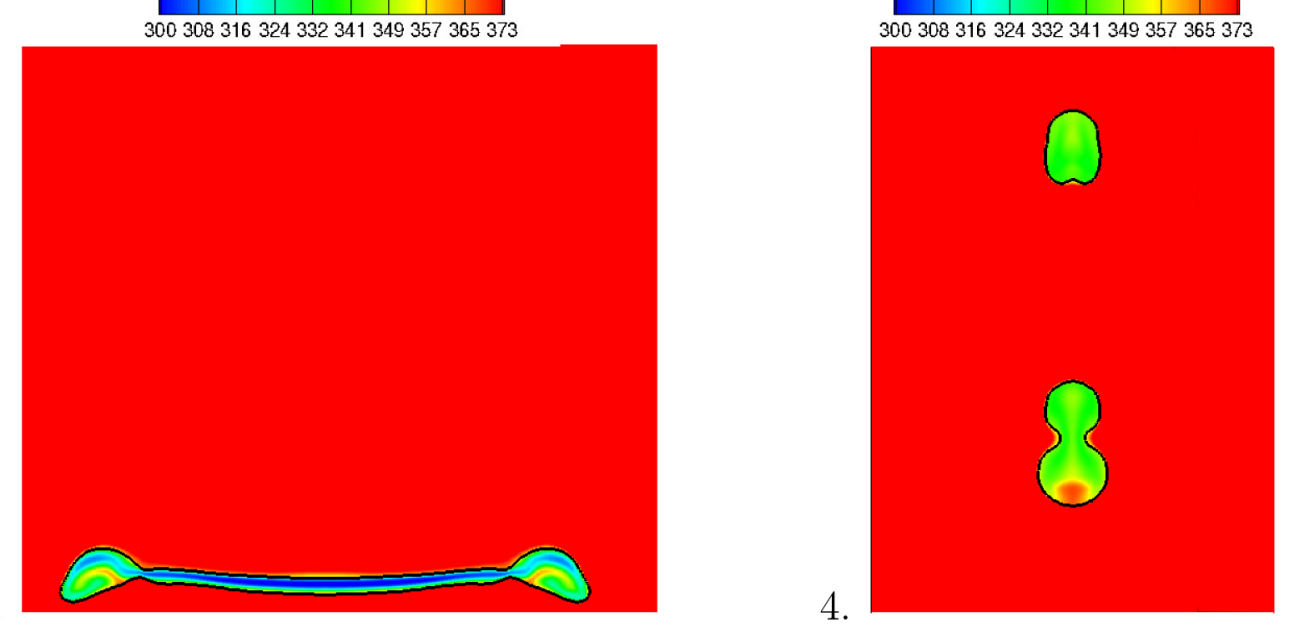

Fig. 20. Temperature field in the liquid phase and interface evolution during the droplet interaction with the hot surface (We $=44.8$ ).

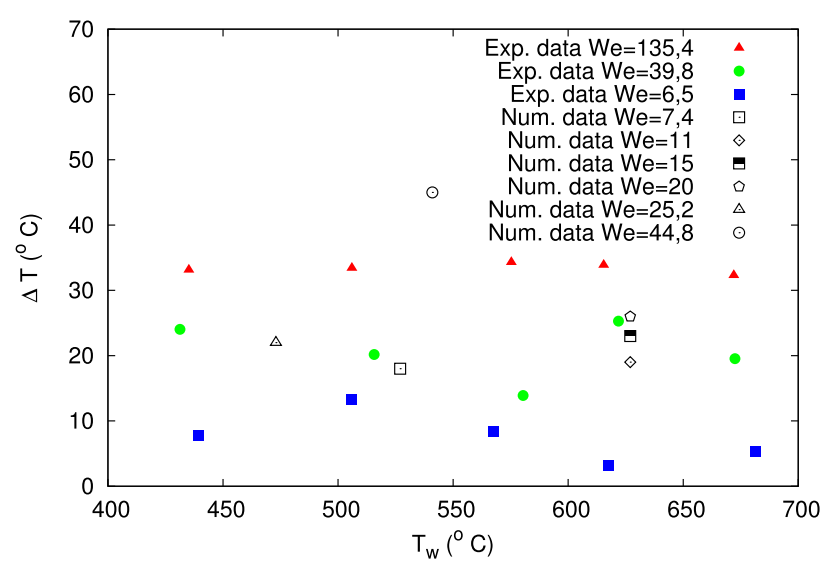

Fig. 21. Droplet heating for different surface temperatures and Weber numbers Comparison between the present experimental results [16] and the present numerical data.

$$
\begin{aligned}
& E_{v}=\frac{1}{E^{*}} \int_{t_{b}}^{t_{a}} \int_{\Gamma} \dot{m} h_{l g} d S d t \\
& E_{\Delta T}=\frac{1}{E^{*}} \int_{t_{b}}^{t_{a}} \int_{\Omega_{l}} \rho C_{p} \partial_{t} \bar{T} d V d t
\end{aligned}
$$

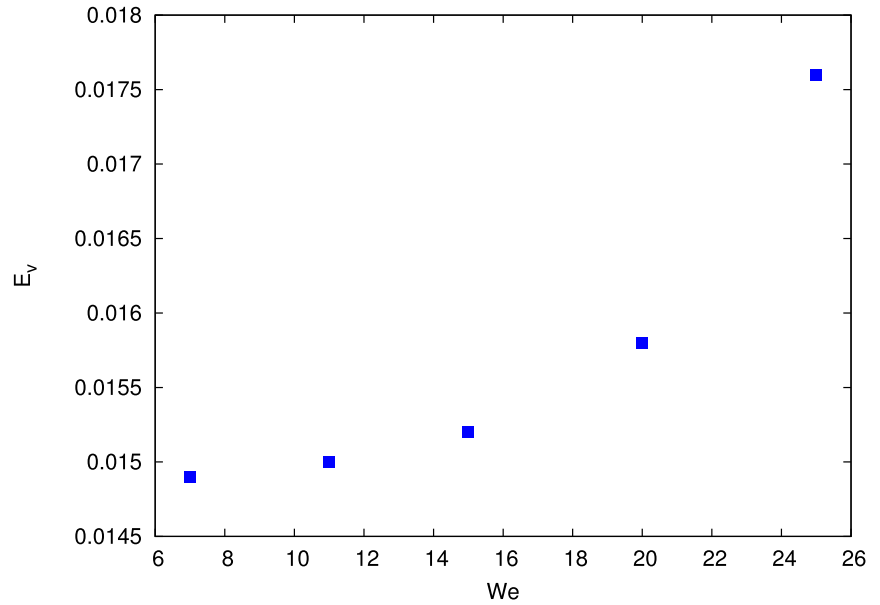

Fig. 22. Dimensionless vaporization energy $E_{v}$ versus the Weber number (Numerical data).

where $E^{*}=\rho_{l} C_{p_{l}} \mathcal{V}\left(T_{\text {sat }}-T_{\text {drop }}\right)$. $t_{b}$ is the impact time, $t_{a}$ the departure time and $T_{\text {drop }}$ the initial average temperature of the droplet. The simulations parameters are given in Table 4.

Fig. 22 shows that under the present conditions, the heat taken by the evaporation increases with the Weber number. It can be 


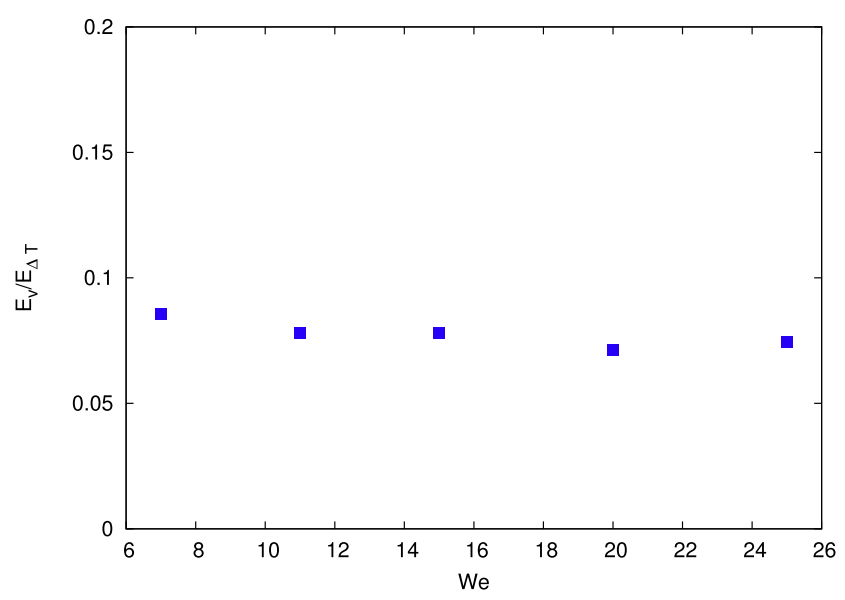

Fig. 23. Energy ratio $E_{v} / E_{\Delta T}$ versus the Weber number (Numerical data).

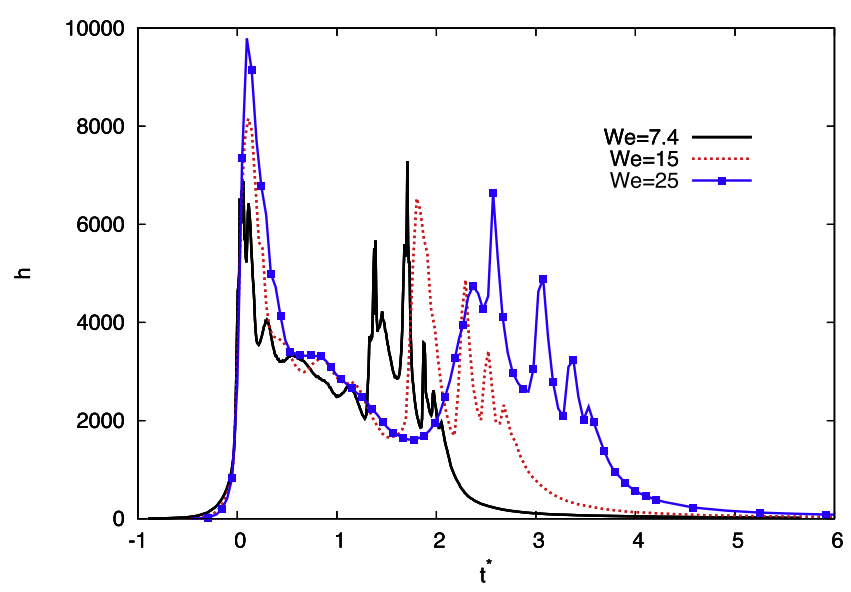

Fig. 24. Time-evolution of the heat transfer coefficient for $W e=7.4, W e=15$, and $W e=25,2$ (Numerical data).

Table 7

Temporal integration of the heat transfer coefficient.

\begin{tabular}{cccc}
\hline$W e$ & 7.4 & 15 & 25.2 \\
\hline $\bar{h}\left(\mathrm{~W} \cdot \mathrm{m}^{-2} \cdot \mathrm{K}^{-1}\right)$ & 2085 & 2137 & 2331 \\
\hline
\end{tabular}

explained by the increase of the maximum spreading diameter (see Fig. 10) and of the maximum droplet surface area with the Weber number. As $\beta_{\max }$ increases, so does the interfacial surface between the droplet and the vapor film, where the heat and mass transfers are the more important. The same tendancy is also reported in [16] where $E_{v}$ and $E_{\Delta T}$ are estimated from the measurements described in Section 3. The energy ratio $E_{v} / E_{\Delta T}$ is displayed in Fig. 23 versus the Weber number. Fig. 23 shows that $E_{v} / E_{\Delta T}$ slightly decreases with We. The heat transfered to the droplet is mostly used to heat up the liquid, and only $10 \%$ is devoted to the liquid evaporation.

Finally, let us focus on the vapor film effectiveness by computing the heat transfer coefficient $h$ and its temporal integration $\bar{h}$ :

$h=\frac{4}{\pi D_{r}^{2}\left(T_{\text {wall }}-T_{\text {sat }}\right)} \int_{0}^{\frac{D_{r}}{2}}-\left.2 k \pi r \frac{\partial T}{\partial z}\right|_{\text {wall }} d r$

$\bar{h}=\frac{1}{t_{\text {res }}^{*}} \int_{0}^{t_{\text {res }}^{*}} h(t) d t$

where $t_{\text {res }}^{*}$, the residence time, is the dimensionless lapse of time that the droplet takes to bounce at the solid surface. The timeevolution of $h$ is reported in Fig. 24 for three different Weber num- bers $(W e=7.4, W e=15$ and $W e=25$ ). The three curves exhibit the same pattern. In each curve, we distinguish two peaks: a major peak at the beginning of the spreading process $\left(t^{*}=0\right)$ and a secondary peak at the receding. The major peak increases with We due to the decrease of the vapor layer thickness in the first stage of the droplet spreading when the Weber number increases. In the three curves, $h \sim 7000 \mathrm{~W} \cdot \mathrm{m}^{-2} \cdot \mathrm{K}^{-1}$ at the secondary peak. The values of the time-average heat transfer coefficient, presented in Table 7, slightly increase with the Weber number. However, they remain close to $\sim 2000 \mathrm{~W} \cdot \mathrm{m}^{-2} \cdot \mathrm{K}^{-1}$. Therefore, for the present simulations, the Weber number has little influence on the heat transfer coefficient.

\section{Conclusions}

In this paper, the impact of droplets onto a hot surface above the Leidenfrost temperature is investigated numerically. The fully resolved simulation of this process is quite challenging since the vapor film is several order of magnitude smaller than the droplet. Also, both boiling and evaporation can occur simultaneously in the vapor film. We develop a novel numerical discretization in the frame of Ghost Fluid/Level Set methods to account for these transient and non-uniform thermodynamic conditions at the liquid/gas interface. Simulations are restricted to moderately small Weber numbers corresponding to the bouncing regime. Therefore, it is an accurate approximation to consider an axisymmetric problem. To validate the simulations, numerical results are also compared to advanced measurements allowing to characterize the droplet deformation, its temperature during its deformation and the heat flux extracted at the solid surface.

Regarding the dynamic behavior of the impinging droplet, a good agreement between numerical and experimental results is observed for the droplet shape evolution, and the onset of satellite droplet. The loss of momentum caused by the viscous dissipation inside the droplet is also well predicted by the simulations.

The present numerical method allows to access all the spatial and temporal scales of the Leidenfrost phenomenon, especially the thickness profile of the vapor layer and the flow within it. During the droplet spreading, the simulations reveal that the vaporization and the motion of the droplet induce an outward radial Poiseuille-like flow, which corroborates the assumption made in $[4,6,9,20,24,38,71]$. However, this assumption is clearly not valid during the receding. Due to the motion of the interface, some surrounding gas is dived into the film which is no longer saturated in vapor.

Finally, after having verified that a reasonable agreement is obtained with the measurements for the droplet heating, an analysis of heat and mass transfer was conducted. Both the heat taken by the evaporation and by the liquid heating appear to increase with the Weber number. Furthermore, in the case of subcooled water droplets injected at $300 \mathrm{~K}$, the main part of the heat transferred to the droplet is used for the heating of the liquid.

\section{Acknowledgements}

The authors gratefully acknowledge funding by ANR (French Research National Agency) in the frame of the IDHEAS (Interaction of Droplets with HEated Surfaces) program.

\section{References}

[1] F. Akao, K. Araki, S. Mori, A. Moriyama, Deformation behaviour of a liquid droplet impinging onto hot metal surface, Trans. ISIJ 20 (1980) 737-743.

[2] K. Anders, N. Roth, A. Frohn, The velocity change of ethanol droplets during collision with a wall analysed by image processing, Exp. Fluids 15 (1993) 9196. 
[3] T.D. Aslam, A partial differential equation approach to multidimensional extrapolation, J. Comput. Phys. 193 (2003) 349-355.

[4] C.T. Avedisian, J. Koplik, Leidenfrost boiling of methanol droplets on hot porous/ceramic surfaces, Int. J. Heat Mass Transfer 30 (1987) 379-393.

[5] J.D. Bernardin, I. Mudawar, The leidenfrost point: experimental study and assessment of existing models, J. Heat Transfer 121 (1999) 894-903.

[6] A.L. Biance, C. Clanet, D. Quéré, Leidenfrost drops, Phys. Fluids 15 (2003) 1632 1637.

[7] J.U. Brackbill, D.B. Kothe, C. Zemach, A continuum method for modeling surface tension, J. Comput. Phys. 100 (1992) 335-354.

[8] J.C. Burton, A.L. Sharpe, R.C.A. van der Veen, A. Franco, S.R. Nagel, Geometry of the vapor layer under a leidenfrost drop, Phys. Rev. Lett. 109 (2012) 074301.

[9] G. Castanet, O. Caballina, F. Lemoine, Drop spreading at the impact in the leidenfrost boiling, Phys. Fluids 27 (2015) 063302.

[10] G. Castanet, O. Caballina, F. Lemoine, Drop spreading at the impact in the leidenfrost boiling, Phys. Fluids 27 (6) (2015).

[11] G. Castanet, P. Dunand, O. Caballina, F. Lemoine, High-speed shadow imagery to characterize the size and velocity of the secondary droplets produced by drop impacts onto a heated surface, Exp. Fluids 54 (2013) 1489.

[12] G.E. Cossali, M. Marengo, M. Santini, Single drop empirical models for spray impact on solid walls: a review, Atomization Sprays 15 (2004) 699-736.

[13] J. Dewitte, Modélisation de l'impact d'un brouillard de gouttes en évaporation et sous pression sur une paroi chauffée (Ph.D. thesis), École Nationale Supérieure de l'Aéronautique et de l'Espace (ENSAE), 2006.

[14] P. Dunand, Etude de l'impact de goutte sur une paroi chaude en régime de Leidenfrost (Ph.D. thesis), EMMA, 2012.

[15] P. Dunand, G. Castanet, M. Gradeck, F. Lemoine, D. Maillet, Heat transfer of droplets impinging onto a wall above the leidenfrost temperature, C.R. Mec. 341 (2013) 75-87.

[16] P. Dunand, G. Castanet, M. Gradeck, D. Maillet, F. Lemoine, Energy balance of droplets impinging onto a wall heated above the leidenfrost temperature, Int. J. Heat Fluid Flow 44 (2013) 170-180.

[17] P. Dunand, G. Castanet, F. Lemoine, A two-planar lif technique to map the temperature of droplets impinging onto a heated wall, Exp. Fluids 52 (4) (2012) 843-856.

[18] A. Esmaeeli, G. Tryggvason, A front tracking method for computations of boiling in complex geometries, Int. J. Multiph. Flow 30 (2004) 1037-1050.

[19] R. Fedkiw, T. Aslam, B. Merriman, S. Osher, A non-oscillatory eulerian approach to interfaces in multimaterial flows, J. Comput. Phys. 152 (1999) 457-492.

[20] H. Fujimoto, Y. Oku, T. Ogihara, H. Takuda, Hydrodynamics and boiling phenomena of water droplets impinging on hot solid, Int. J. Multiph. Flow 36 (2010) 620-642.

[21] Y. Ge, L.S. Fan, Three-dimensional simulation of impingement of a liquid droplet on a flat surface in the leidenfrost regime, Phys. Fluids 17 (2005) 027104.

[22] F. Gibou, L. Chen, D. Nguyen, S. banerjee, A level set based sharp interface method for the multiphase incompressible navier-stokes equations with phase change, J. Comput. Phys. 222 (2007) 536-555.

[23] F. Gibou, C. Min, R. Fedkiw, High resolution sharp computational methods for elliptic and parabolic problems in complex geometries, J. Sci. Comput. 54 (2013) 369-413.

[24] B.S. Gottfried, K.J. Bell, Film boiling of spheroidal droplets, Ind. Eng. Chem. Fundam. 5 (1966) 561-568.

[25] B.S. Gottfried, C.J. Lee, K.J. Bell, The leidenfrost phenomenon: film boiling of liquid droplets on a flat plate, Int. J. Heat Mass Transfer 9 (1966) 1167-1188.

[26] M. Gradeck, N. Seiler, P. Ruyer, D. Maillet, Heat transfer for leidenfrost drops bouncing onto a hot surface, Exp. Thermal Fluid Sci. 47 (2013) 14-25.

[27] V. Groendes, R. Mesler, Measurement of transient surface temperatures beneath leidenfrost water drops, Proceedings of the 7th International Heat Transfer Conference (Munich, Germany), vol. 21, 1982, pp. 131-136.

[28] S. Hardt, F. Wondra, Evaporation model for interfacial flows based on a continuum-field representation of the source terms, J. Comput. Phys. 227 (2008) 5871-5895.

[29] D.J.E. Harvie, D.F. Fletcher, A hydrodynamic and thermodynamic simulation of droplet impact on hot surfaces, part I: theoretical model, Int. J. Heat Mass Transfer 44 (2001) 2633-2642.

[30] D.J.E. Harvie, D.F. Fletcher, A hydrodynamic and thermodynamic simulation of droplet impact on hot surfaces, part II: validation and application, Int. J. Heat Mass Transfer 44 (2001) 2643-2659.

[31] N. Hatta, H. Fujimoto, K. Kinoshita, H. Takuda, Experimental study of deformation mechanism of a water droplet impinging on hot metallic surfaces above the leidenfrost temperature, ASME. J. Fluids Eng. 119 (1997) 692-699.

[32] N. Hatta, H. Fujimoto, H. Takuda, K. Kinoshita, O. Takahashi, Collision dynamics of a water droplet impinging on a rigid surface above the leidenfrost temperature, ISIJ Int. 35 (1994) 50-55.

[33] R.W. Houim, K.K. Kuo, A ghost fluid method for compressible reacting flows with phase change, J. Comput. Phys. 235 (2013) 865-900.

[34] G. Huber, S. Tanguy, J.C. Béra, B. Gilles, A time splitting projection scheme for compressible two-phase flows. application to the interaction of bubbles with ultrasound waves, J. Comput. Phys. 301 (2015) 439-468.

[35] S. Inada, Y. Miyasaka, K. Nishida, Transient heat transfer for a water drop impinging on a heated surface, Bulletin of JSME 28 (1985) 2675-2681.

[36] D. Juric, G. Tryggvason, Computations of boiling flows, Int. J. Multiph. Flow 24 (1998) 387-410.
[37] M. Kang, R. Fedkiw, X.D. Liu, A boundary condition capturing method for multiphase incompressible flow, J. Comput. Phys. 15 (2000) 323-360.

[38] A. Karl, A. Frohn, Experimental investigation of interaction processes between droplets and hot walls, Phys. Fluids 12 (1999) 785-796.

[39] C. Kunkelmann, P. Stephan, Numerical simulation of the transient heat transfer during nucleate boiling of refrigerant hfe-7100, Int. J. Fluid Refrig. 33 (2010) $1221-1228$.

[40] B. Lalanne, L. Rueda-Villegas, S. Tanguy, F. Risso, On the computation of viscous terms for incompressible two-phase flows with level set/ ghost fluid method, J. Comput. Phys. 301 (2015) 289-307.

[41] J. Lee, G. Son, A level-set method for analysis of particle motion in an evaporating microdroplet, Numer. Heat Transfer, Part B 67 (2015) 25-46.

[42] J.G. Leidenfrost, On the fixation of water in diverse fire, Int. J. Heat Mass Transfer 9 (1966) 1153-1166.

[43] M. Lepilliez, E.R. Popescu, F. Gibou, S. Tanguy, On two-phase flow solvers in irregular domains with contact line, J. Comput. Phys. (2016), http://dx.doi.org/ 10.1016/j.jcp.2016.06.013.

[44] X. -D Liu, R. Fedkiw, M. Kang, A boundary condition capturing method for poisson's equation on irregular domains, J. Comput. Phys. 160 (2000) 151-178.

[45] C. Ma, D. Bothe, Numerical modeling of thermocapillary two-phase flows with evaporation using a two-scalar approach for heat transfer, J. Comput. Phys. 233 (2013) 552-573.

[46] D. Nguyen, R. Fedkiw, M. Kang, A boundary condition capturing method for incompressible flame discontinuities, J. Comput. Phys. 172 (2001) 71-98.

[47] N. Nikolopoulos, A. Theodorakakos, G. Bergeles, A numerical investigation of the evaporation process of a liquid droplet impinging onto a hot substrate, Int. J. Heat Mass Transfer 50 (2006) 303-319.

[48] J. Papac, F. Gibou, C. Ratsch, Efficient symmetric discretization for the poisson, heat and stefan-type problems with robin boundary conditions, J. Comput. Phys. (2009).

[49] Y.M. Qiao, S. Chandra, Boiling of droplet on a hot surface in low gravity, Int. J. Heat Mass Transfer 39 (1995) 1379-1393.

[50] M. Renksizbulut, R. Nafziger, X. Li, A mass transfer correlation for droplet evaporation in high-temperature flows, Chem. Eng. Sci. 46 (1991) $2351-$ 2358.

[51] I.V. Roisman, E. Berberovic, C. Tropea, Inertia dominated drop collisions. i. on the universal flow in the lamella, Phys. Fluids 21 (5) (2009).

[52] I.V. Roisman, C. Planchette, E. Lorenceau, G. Brenn, Binary collisions of drops of immiscible liquids, J. Fluid Mech. 690 (2012) 512-535.

[53] I.V. Roisman, R. Rioboo, C. Tropea, Normal impact of a liquid drop on a dry surface: model for spreading and receding, Proc. R. Soc. London A: Math. Phys. Eng. Sci. 458 (2022) (2002) 1411-1430.

[54] L. Rueda-Villegas, R. Alis, M. Lepilliez, S. Tanguy, A ghost fluid/level set method for boiling flows and liquid evaporation: Application to the leidenfrost effect, J. Comput. Phys. 316 (2016) 789-813.

[55] Y. Sato, B. Niceno, A sharp-interface phase change model for a massconservative interface tracking method, J. Comput. Phys. 249 (2013) 127-161.

[56] Y. Sato, B. Niceno, A depletable micro-layer model for nucleate pool boiling, J. Comput. Phys. 300 (2015) 20-52.

[57] J. Schlottke, B. Weigand, Direct numerical simulation of evaporating droplets, J. Comput. Phys. 227 (2008) 5215-5237.

[58] B. Sobac, A. Rednivov, S. Dorbolo, P. Colinet, Leidenfrost effect: Accurate drop shape modeling and refined scaling laws, Phys. Rev. E 90 (2014) 053011.

[59] G. Son, V.K. Dhir, Numerical simulation of film boiling near critical pressures with a level set method, J. Heat Tansfer 120 (1998).

[60] G. Son, V.K. Dhir, N. Ramanujapu, Dynamics and heat transfer associated with a single bubble during nucleate boiling on a horizontal surface, J. Heat Tansfer 121 (1999) 623-631.

[61] D. B. Spalding. Combustion and mass transfer, Pergamon, 1979.

[62] M. Sussman, P. Smereka, S. Osher, A level set approach for computing solutions to incompressible two-phase flow, J. Comput. Phys. 114 (1994) 146-159.

[63] M. Sussman, K.M. Smith, M.Y. Hussaini, m. Ohta, R. Zhi-Wei, A sharp interface method for incompressible two-phase flow, J. Comput. Phys. 221 (2007) 469505.

[64] S. Tanguy, T. Ménard, A. Berlemont, A level set method for vaporizing twophase flows, J. Comput. Phys. 221 (2007) 837-853.

[65] S. Tanguy, M. Sagan, B. Lalanne, F. Couderc, C. Colin, Benchmarks and numerical methods for the simulation of boiling flows, J. Comput. Phys. 264 (2014) 1-22.

[66] T. Ueda, T. Enomoto, M. Kanetsuki, Heat transfer characteristics and dynamic behavior of saturated droplets impinging on a heated vertical surface, Jpn. Soc. Mech. Eng. 22 (1979) 724-732.

[67] L.H.J. Wachters, N.A.J. Westerling, The heat transfer from a hot wall to impinging water drops in the spheroidal state, Chem. Eng. Sci. 21 (1966) 1047-1056.

[68] S.C. Yao, K.Y. Cai, The dynamics and leidenfrost temperature of drops impacting on a hot surface at small angles, Exp. Thermal Fluid Sci. 1 (1988) 363-371.

[69] A.L. Yarin, Drop impact dynamics: splashing, spreading, receding, bouncing, Annu. Rev. Fluid Mech. 38 (2005) 159-192.

[70] A.L. Yarin, D.A. Weiss, Impact of drops on solid surfaces: self-similar capillary waves, and splashing as a new type of kinematic discontinuity, J. Fluid Mech. 283 (1) (1995) 141-173.

[71] S. Zhang, G. Gogos, Film evaporation of a spherical droplet over a hot surface: fluid mechanics and heat/mass transfer analysis, J. Fluid Mech. 222 (1990) 543-563. 